\title{
Las emociones en la enseñanza de las ciencias*
}

\section{Emotions in Science teaching}

Vicente Mellado, A. Belén Borrachero, María Brígido, Lina V. Melo, M. Antonia Dávila, Florentina Cańada, M. Carmen Conde, Emilio Costillo, Javier Cubero, Rocío Esteban, Guadalupe Martínez, Constantino Ruiz, Jesús Sánchez Departamento de Didáctica Ciencias Experimentales y Matemáticas. Universidad de Extremadura. vmellado@unex.es

Andoni Garritz

Facultad de Quimica, Universidad Nacional Autónoma de México. México DF.

Lucía Mellado

Departamento de Economía de La Empresa y Contabilidad. Facultad Ciencias Económicas y Empresariales. UNED.

Bartolomé Vázquez, Roque Jiménez

Departamento de Didáctica de las Ciencias y Filosofía. Universidad de Huelva.

M. Luisa Bermejo

Departamento de Psicología y Antropología. Universidad de Extremadura.

RESUMEN • En la primera parte del artículo, se incide en la importancia del estudio de las emociones desde la didáctica de las ciencias, así como en la necesidad de establecer programas de intervención metacognitivos y metaemocionales, tanto en el aprendizaje como en la formación del profesorado, para que alumnos y profesores puedan conocer sus emociones, controlarlas y autorregularlas. En la segunda parte, nos centramos en las emociones en el conocimiento didáctico del contenido del profesorado de ciencias, incluyendo algunos resultados tanto del diagnóstico emocional del profesorado, según distintas variables, como del programa de intervención de la Universidad de Extremadura.

PALABRAS CLAVE: emociones; enseñanza de las ciencias; conocimiento didáctico del contenido.

ABSTRACT $•$ The first part of this communication highlights the importance of studying emotions in the context of science teaching. It also examines the need for programs of meta-emotional and metacognitive intervention in learning and teacher education that are aimed at both the future teachers' and their pupils' gaining awareness of, and then controlling and self-regulating, their emotions. The second part focuses on the role emotions play in science teachers' pædagogical content knowledge, with the presentation of some results concerning the University of Extremadura's intervention program and the emotional diagnosis of teachers.

KEYWORDS: emotions; science teaching; pedagogical content knowledge.

Fecha de recepción: mayo 2014• Aceptado: septiembre 2014

*Una primera versión de este artículo fue presentada por Vicente Mellado Jiménez en la conferencia de clausura del IX Congreso Internacional sobre Investigación en Didáctica de las Ciencias, celebrado en Girona del 9 al 12 de septiembre del 2013. 


\section{INTRODUCCIÓN}

En las cuatro últimas décadas, la didáctica de las ciencias ha tratado de encontrar explicaciones racionales a los procesos de enseñanza-aprendizaje de las ciencias experimentales y, desde su constitución como disciplina emergente, se ha consolidado como dominio específico de conocimiento, existiendo una verdadera comunidad de profesores e investigadores en el área (Aduriz-Bravo e Izquierdo, 2002; Gil et al., 2000). Una prueba de ello es la publicación de cuatro Handbook internacionales y más de 100 revistas especializadas en el área, de las cuales más de 20 están ya incluidas en el Journal Citation Report, entre ellas Enseñanza de las Ciencias.

En la investigación en didáctica de las ciencias, se ha incidido sobre todo en los factores cognitivos de enseñanza-aprendizaje de las distintas materias de ciencias, descuidando el dominio afectivo y emocional. La Psicología ya lleva tratando las emociones desde hace ańos, pero como los propios psicólogos reconocen (Manassero, 2013), las emociones han estado excluidas durante siglos de la Filosofía, de la Psicología y no digamos de la ciencia o de la didáctica de las ciencias. A pesar de los avances epistemológicos de las distintas orientaciones filosóficas sobre la naturaleza de la ciencia, en la enseñanza de las ciencias ha imperado una abusiva orientación positivista que, de hecho, en la mayoría de los casos ha excluido los factores sociales, culturales o afectivos, tildados como impropios o acientíficos por oponerse a la objetividad de la ciencia, aunque sean didácticamente valiosos (Vázquez y Manassero, 2007).

Los cuatro Handbooks internacionales de didáctica de las ciencias no dedican ninguna sección específica a las emociones, aunque este aspecto es tratado en algunos capítulos, generalmente relacionándolos con las actitudes más específicamente que con las emociones. Los estudios sobre las actitudes de los estudiantes hacia las ciencias nos dejan unos preocupantes resultados (Pérez y de Pro, 2013; Vázquez y Manassero, 2008 y 2011):

- El interés de los escolares hacia la ciencia comienza pronto, pero es decreciente de primaria a secundaria.

- Consideran la ciencia escolar aburrida y poco relevante para sus vidas.

- Las actitudes influyen en la elección de asignaturas y de estudios universitarios.

- Hay diferencias en las actitudes de chicos y chicas según las materias: los chicos están más a favor de temas de Física, Química y Tecnología, y las chicas más a favor de temas de Salud y de Ciencias de la vida. Hay estereotipos de género asociados al rol profesional (Gutiérrez y Luengo, 2003) que transmiten que los hombres poseen mayores capacidades para el desarrollo de tareas científicas.

Desde la inicial línea de las actitudes, el estudio de las emociones en el proceso de enseñanza-aprendizaje de las ciencias se abre paso en congresos y revistas de didáctica de las ciencias, y cada vez son más frecuentes los trabajos centrados en esta temática (Abrahams, 2009; Bellocchi et al., 2013; Dos Santos y Mortimer, 2003; Hugo et al., 2013; Marbá y Márquez, 2010, Mellado et al., 2013; Otero, 2006; Ritchie et al., 2011; Schutz y Zembylas, 2011; Vázquez y Manassero, 2007; Zembylas, 2002 y 2007).

\section{MARCO TEÓRICO}

\section{Las emociones en la didáctica de las ciencias}

Desde el constructivismo, marco teórico mayoritario en las últimas décadas en didáctica de las ciencias en el estudio de las ideas alternativas del alumnado y del cambio conceptual, también se está realizando un acercamiento hacia las emociones. El cambio conceptual es tanto cognitivo como afectivo (Thagard, 2008) y los profesores que ignoran los aspectos afectivos del aprendizaje pueden limitar el 
cambio conceptual en sus alumnos. Pintrich et al. (1993) ya cuestionaban el «cambio frío», y defendían la importancia de la motivación y de las emociones como factores determinantes en el aprendizaje de las ciencias. Desde una perspectiva sociocultural de la didáctica de las ciencias, Tobin (2010: 303) seńala que en el aprendizaje de las ciencias «las emociones actúan como un pegamento social que interconecta intereses y acciones individuales y colectivas». Los estados emocionales positivos favorecen el aprendizaje de las ciencias y el compromiso de los estudiantes como aprendices activos, mientras que los negativos limitan la capacidad de aprender (Olitsky y Milne, 2012; Vázquez y Manassero, 2007). Esta podría ser una de las causas por la que algunos alumnos superdotados, con un alto coeficiente intelectual cognitivo, fracasen en sus estudios.

El estudio de las emociones no es un tema nuevo en las ciencias experimentales. Darwin publicó en 1872 el libro La expresión de las emociones en el hombre y en los animales, una obra de éxito editorial inmediato, ya que Darwin era famoso porque trece años antes había publicado El origen de las especies, aunque el éxito fue efímero, porque la obra se olvidó y apenas tuvo influencia en la investigación científica. En esta obra ya se refleja que las emociones son una forma básica de regular la vida, forman parte de nuestra estructura evolutiva, cumplen una función adaptativa de nuestro organismo a lo que nos rodea, han sido importantes en la supervivencia de nuestra propia especie y no siempre están controladas por la razón.

Aún hoy muchos profesores e investigadores consideran que las emociones están al margen de la racionalidad científica y, por tanto, hay que excluirlas de la investigación educativa. Pero eso sería reconocer nuestra incapacidad para comprender las zonas oscuras del pensamiento y de la conducta. En cierto modo sería como volver al oscurantismo y a la superstición, con los que tanto ha tenido que luchar el conocimiento científico a lo largo de la historia.

Como señala Hargreaves (1998: 558): «Las emociones están en el corazón de la enseñanza». Los sentimientos y las emociones tienen un papel vital en el desarrollo del aprendizaje, ya que el mundo subjetivo y emocional que cada persona desarrolla sobre la realidad exterior da sentido a las relaciones y hace comprender el lugar propio que ocupamos en un mundo más amplio. Actualmente se considera que lo cognitivo configura lo afectivo y lo afectivo lo cognitivo, siguiendo la teoría de los moldes cognitivo-afectivos de Hernández (2002), y la idea de la enseñanza-aprendizaje como una práctica emocional en la que intervienen procesos cognitivos y afectivos es aceptada por investigadores y educadores (Hargreaves, 1996 y 1998). En nuestro ámbito, la popularidad de autores como Goleman (1996), Marina (2004) o Punset (2010) han contribuido a despertar el interés por las emociones. Sin embargo hay que estar alerta porque la historia nos enseña cómo, desde las emociones, se pueden cometer las mayores barbaridades individuales y colectivas, si no se las gobierna desde la razón y la ética (Camps, 2012).

La investigación sobre los emociones cubre aspectos generales, tratados desde otras disciplinas científicas, y otros específicos relacionados con los contenidos disciplinares de las diferentes materias que deben ser objeto de estudio desde las didácticas específicas (Vázquez y Manassero, 2007), ya que las emociones también están ligadas a la enseñanza-aprendizaje de conocimientos concretos (Dolan, 2002; Garritz y Ortega-Villar, 2013).

Otero (2006) señala que no hay una acción humana sin una emoción que la fundamente y la haga posible. La emoción es fundamental en la toma de decisiones (Angie et al., 2011; Damasio, 1996), algo que profesores y alumnos tienen que hacer constantemente en clase. Para los alumnos, la toma de decisiones se vuelve especialmente importante cuando, al final de la educación obligatoria, tienen que decidir la orientación de sus futuros estudios. La disminución de las carreras relacionadas con la ciencia ha llegado a ser algo preocupante en muchos países (Rocard et al., 2007). Esto puede estar relacionado con el contexto emocionalmente adverso que rodea el aprendizaje científico y con el hecho de que muchos alumnos tienen que decidir sobre la dirección futura de sus estudios después de haber tenido 
un aprendizaje de las ciencias centrado en la transmisión abstracta de conceptos, con poca relevancia para sus vidas fuera de la escuela y con el que no han conseguido disfrutar (Vázquez y Manassero, 2007 y 2011).

Si, como señalan Bisquerra y Pérez (2007), los conocimientos académicos se aprenden mejor si los alumnos tienen competencias emocionales, es fundamental formar profesores emocionalmente competentes que sepan diagnosticar y autorregular sus emociones a través de programas de intervención que incluyan tanto lo cognitivo como lo afectivo. Pero en un tema tan complejo es necesario abrirnos a otros campos y trabajar en equipos interdisciplinares. Necesitamos integrar las aportaciones de la propia didáctica de las ciencias con las que se realizan desde otras didácticas específicas, la ciencia básica, la neurociencia, la psicología, la sociología, la pedagogía, la ética y otras más. Esto nos obliga a tener en cuenta no solo las emociones o las actitudes, sino otros muchos constructos analizados desde otros campos como la autoeficacia, el autoconcepto, las inteligencias múltiples de Gardner (2005), etc.

\section{Conceptualización de las emociones}

Existe una gran variedad de taxonomías para referirse al dominio afectivo, a las emociones, a los sentimientos, al estado de ánimo, etcétera. (Manassero, 2013). Las concepciones/creencias, las actitudes, las emociones o el dominio afectivo han tenido distinta interpretación en la literatura. Desde la didáctica de las matemáticas, McLeod (1989) incluye como componentes del dominio afectivo a las creencias, las actitudes y las emociones. Koballa y Glynn (2007) consideran que en didáctica de las ciencias las creencias se han asociado generalmente al dominio cognitivo, mientras las actitudes lo han sido al afectivo y emocional. Vázquez y Manassero (2007) se refieren al ámbito emotivo-actitudinal y consideran que el componente afectivo es el rasgo más relevante de la actitud. También en didáctica de las ciencias, Dos Santos y Mortimer (2003) consideran que lo afectivo es más general e incluye a las emociones, a los sentimientos y al estado de ánimo. Damasio (2010) distingue entre las emociones como percepciones que se acompañan de ideas y modos de pensamiento, y los sentimientos como percepciones de lo que hace el cuerpo mientras se manifiesta la emoción. En nuestros estudios, nos referiremos fundamentalmente a las emociones, aunque en ocasiones hagamos referencia genérica a la dimensión afectiva o a los sentimientos.

Son muchas las definiciones que han ido apareciendo a lo largo de los años para referirse a las emociones. Una de las más asumidas en nuestro ámbito es la de Bisquerra (2000), quien señala que las emociones son reacciones a la información recibida de nuestro entorno, cuya intensidad depende de las evaluaciones subjetivas que realizamos y en las que tienen gran influencia los conocimientos previos y las creencias. En definitiva, una emoción depende de lo que es importante para nosotros. Damasio (2010) añade que las emociones no solo son reacciones a los estímulos del presente, sino que también se producen por el recuerdo o la evocación de hechos sucedidos en el pasado o por el anticipo de posibles situaciones futuras.

En resumen, una emoción es una reacción subjetiva a los estímulos del ambiente acompañada de cambios orgánicos (fisiológicos y endocrinos) de origen innato, pero influidos por la experiencia individual y social. Darwin estudió la expresión de las emociones en personas y animales y asumió su origen genético como un refuerzo a la teoría de la evolución. En cambio, otros autores dan más importancia a lo adquirido a través de la experiencia individual y social. Asumimos que la emoción tiene una parte psicobiológica (Damasio, 2010; Mora, 2008), pero es también una construcción social (White, 1993) interconectada con el contexto y la cultura (Lasky, 2000). Como dice Damasio (2010: 194): «Aunque las emociones son automáticas, no hay determinismo, puesto que hay una considerable personalización de las respuestas emocionales en relación con el estímulo, por la causa, la cultura, etcétera». 
Hay muchas taxonomías no coincidentes para clasificar las emociones. Hay emociones primarias o básicas, con un origen genético, y emociones sociales, que se construyen socialmente y dependen más del contexto (Camps, 2012). Para Manassero (2013), las emociones básicas son entidades psicofisiológicas, son observaciones conductuales que aparecen en todas las culturas y se caracterizan por un rápido comienzo, corta duración y ocurrencia espontánea. Entre las básicas, primarias o de fondo se encuentran el miedo, la aversión, la ira, la tristeza, la alegría y la sorpresa, aunque esta última Damasio (2005) la ve también con un cierto contenido social junto con las sociales como la vergüenza, la culpabilidad, los celos, la simpatía, la turbación, el orgullo, la envidia, la gratitud, la admiración, la indignación y el desdén. Damasio (2010) añade las emociones de forma (por ejemplo, el entusiasmo y el desaliento), que pueden desencadenarse por la reflexión de una situación pasada o por la consideración de una posible situación futura.

$\mathrm{Si}$ atendemos a los estudios sobre sus efectos, las emociones también pueden clasificarse en positivas (la alegría, el orgullo, la gratitud, etcétera), negativas (el miedo, la ira, la aversión, la culpa, etcétera) o neutras como la sorpresa. Para Fernández-Abascal et al. (2001), las emociones positivas implican sentimientos agradables, con duración temporal corta y movilizan escasos recursos para su afrontamiento; en cambio, las emociones negativas implican sentimientos desagradables y movilizan muchos recursos para su afrontamiento; las emociones neutras no producen intrínsecamente reacciones ni agradables ni desagradables y tienen como finalidad el facilitar la aparición de posteriores estados emocionales.

Darwin (1872) ya señalaba la existencia de emociones estimulantes y emociones depresoras. Para Manassero (2013: 8): «Las emociones sirven para motivar, organizar, dirigir y activar conductas, pero también pueden bloquear o interrumpir otras conductas». Del mismo modo, Camps (2012: 13) considera que «hay emociones que nos incitan a actuar, otras nos llevan a escondernos o a huir de la realidad». En esta línea Díaz y Flores (2001) y López-Ramírez (2009) clasifican las emociones en dos ejes de coordenadas: en el vertical las agradables-desagradables y en el horizontal las contrapuestas de relajaciónexcitación. Entre las clasificadas como excitantes están, entre otras, el vigor, la diversión, la tensión, el entusiasmo, la duda, la satisfacción, la ira, la alegría, el odio, el valor o la aversión. En sus opuestas están el agotamiento, el aburrimiento, la apatía, la certeza, la frustración, la tristeza, o el miedo.

Desde la psicología y la pedagogía se han propuesto distintos marcos teóricos para el estudio de las emociones, aunque por centrarse en la didáctica de las ciencias destacamos la síntesis que realizan Hugo et al. (2013) de las distintas orientaciones en cuatro perspectivas: la neurobiológica (Damasio, 1996), que vincula la mente con lo orgánico y las emociones con la acción; la socioconstructivista (Hargreaves, 1998; Zembylas, 2002), que destaca las relaciones sociales, culturales e ideológicas y su expresión; la sociocognitiva (Dos Santos y Mortimer, 2003), que analiza las emociones como mediadoras entre el pensamiento y el contexto social, enfatizando los factores motivacionales para el aprendizaje y las atribuciones de causa-efecto (Weiner, 1986), y la interaccionista, que busca más la integración que las dicotomías y lo holístico entre lo individual y lo social.

\section{Las emociones en el conocimiento didáctico del contenido del profesorado de ciencias experimentales}

Shulman (1986) consideraba que además del conocimiento de la materia y del conocimiento psicopedagógico general, entre otros, los profesores desarrollan un conocimiento específico sobre la forma de enseñar su materia al que denominó el conocimiento didáctico del contenido (CDC). Este conocimiento es elaborado de forma personal por los profesores en la práctica de la enseñanza, constituye un cuerpo de conocimientos que distingue a la enseñanza como profesión y es una forma de razonamiento y acción didáctica por la cual los profesores transforman un contenido dado en representaciones comprensibles para sus estudiantes. En el CDC se incluyen, para los tópicos más regularmente enseñados 
en el área temática del profesor, «las formas más útiles de representación de estas ideas; las analogías, ilustraciones, ejemplos, explicaciones y demostraciones más poderosos; en pocas palabras, las formas de representación y formulación del tema que lo hace comprensible a otros» (Shulman, 1987: 9). El CDC tiene mucho en común con lo que Porlán et al. (2010) denominan el conocimiento práctico profesional.

Desde entonces se han realizado numerosas formulaciones y el CDC se ha convertido en uno de los temas relevantes de la investigación en didáctica de las ciencias (Acevedo, 2009; Mellado, 2011; Melo et al., 2013). En síntesis, podemos distinguir tres fuentes del CDC en las distintas etapas de la vida del profesor: sus propios antecedentes escolares, la formación inicial y la experiencia profesional (Friedrichsen et al., 2009).

En la formación inicial, el conocimiento académico base necesario para la enseñanza (conocimiento del contenido, psicopedagógico general, de didáctica de las ciencias, etcétera) se integra y transforma en el CDC durante la práctica de la enseñanza, enmarcado en el contexto particular en el que se realiza la enseñanza. En la formulación más utilizada de Magnusson et al. (1999), el CDC del profesor tiene cinco componentes, todos ellos interrelacionados y centrados en la materia específica de enseñanza: la visión y propósitos sobre la enseñanza, el conocimiento sobre el currículo, sobre las estrategias de enseñanza, sobre los estudiantes y el aprendizaje, y sobre la evaluación. Para Friedrichsen et ál. (2009), la visión y los propósitos sobre la enseñanza tienen un estatus diferente a los otros cuatro componentes, ya que también condicionan e influyen en el conocimiento académico y en el propio proceso de transformación e integración en el CDC.

Inicialmente las emociones y el dominio afectivo no habían sido consideradas como parte del CDC. Sin embargo, en los últimos años numerosos autores consideran que las emociones sobre el proceso de enseñanza-aprendizaje de cada materia deben formar parte del CDC del profesor (Garritz, 2010; Garritz y Ortega-Villar, 2013; Kind, 2009; McCaughtry, 2005; Padilla y van Driel, 2012; Park y Oliver, 2008; Zembylas, 2007). En los estudios de Rosiek y Beghetto (2011), los profesores raramente se plantean dicotomías entre lo cognitivo y lo afectivo, sino que interpretan el proceso de aprendizaje de sus alumnos como un tejido integrado entre emoción, contenidos, la historia personal del estudiante, el contexto y la relación alumno-profesor. Recientemente el propio Shulman (2012) reconoce que el olvido de la parte afectiva fue una de las debilidades de las formulaciones iniciales sobre el CDC y defiende que las emociones deberían incorporarse a este.

En la actualidad, sigue siendo materia de debate si las emociones tienen el mismo estatus que los propósitos y actúan como una lente a través de la que el profesor percibe los demás componentes del CDC y su propio proceso de desarrollo o si, como defienden Garritz y Ortega-Villar (2013), las emociones son el sexto componente perdido del CDC (figura 1). 


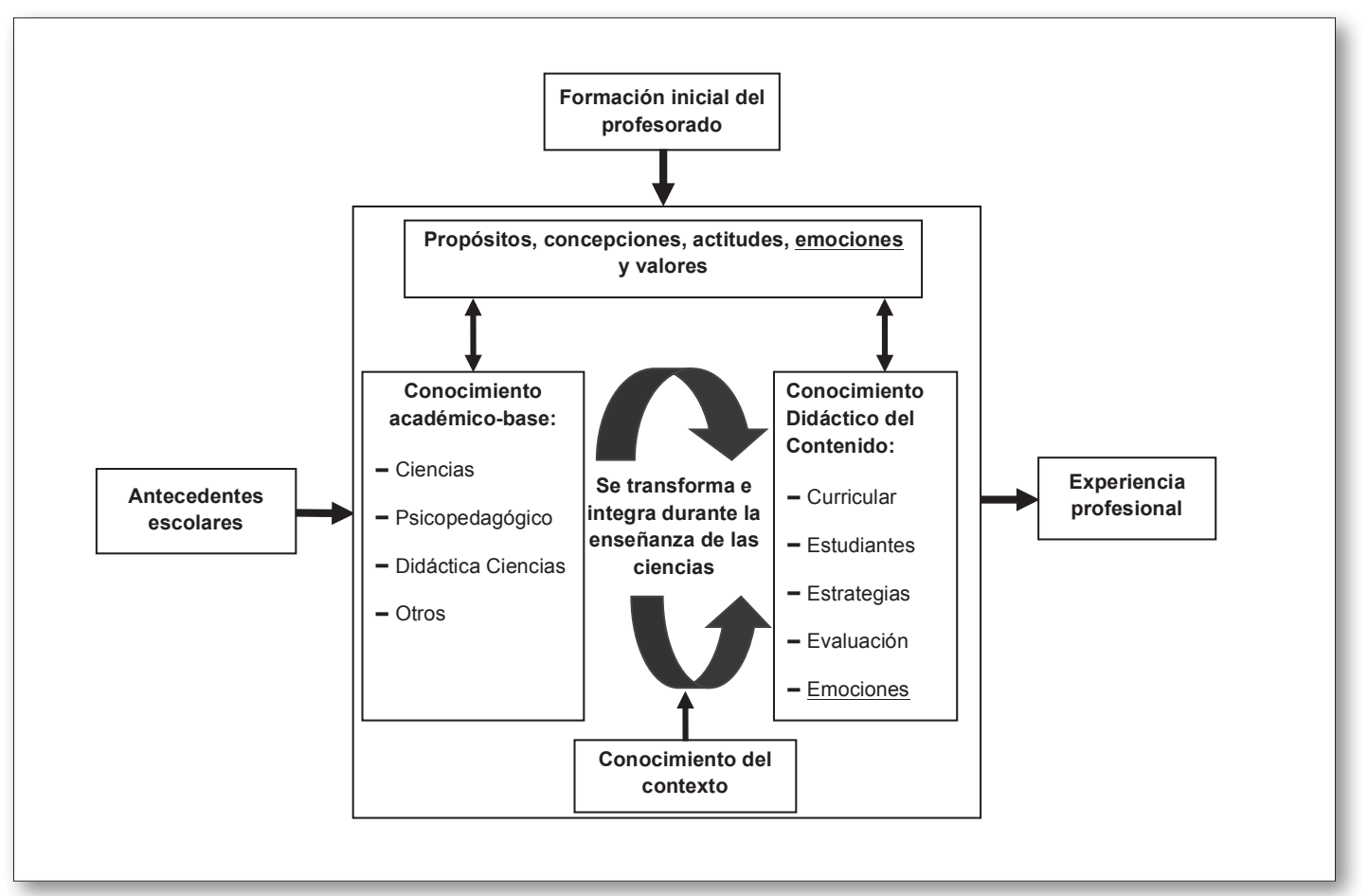

Fig. 1. El conocimiento didáctico del contenido tiene su origen en los antecedentes escolares, se inicia en la formación inicial y se desarrolla en la experiencia profesiona

\section{ALGUNOS RESULTADOS}

El programa de investigación que estamos llevando a cabo en la Universidad de Extremadura abarca dos líneas: por un lado, el diagnóstico y la intervención de las emociones en el aprendizaje de las ciencias y la Tecnología en el alumnado de secundaria y de universidad, analizando las causas de las emociones en las distintas materias de ciencias y Tecnología de la ESO, su relación con el tipo de contenidos, con las estrategias de enseñanza, con las actividades de aprendizaje y con el perfil de los alumnos, así como con otras variables como la autoeficacia, el autoconcepto y la autorregulación, y por otro, el diagnóstico y la intervención de las emociones en ciencias y Tecnología en la formación y el desarrollo profesional del profesorado de primaria y secundaria de estas materias.

Aunque ambas líneas están interrelacionadas y son complementarias, a continuación nos centraremos fundamentalmente en la segunda de ellas, sobre las emociones en la formación y el desarrollo profesional del profesorado de ciencias de primaria y secundaria, tomando como constructo integrador el conocimiento didáctico del contenido.

\section{El recuerdo de las emociones hacia el aprendizaje de las ciencias de los profesores en formación}

Los antecedentes escolares son una de las fuentes del CDC, ya que los profesores en formación tienen unas creencias, actitudes y emociones hacia la enseñanza-aprendizaje de las distintas asignaturas. La enseñanza se distingue de otras profesiones en que cuando los futuros profesores comienzan su etapa de formación universitaria tienen ideas, concepciones, actitudes, valores y emociones sobre la ciencia y sobre la forma de aprenderla y enseñarla, fruto de los años que han pasado como escolares, y asumen o rechazan los roles de los profesores que tuvieron en su etapa escolar y universitaria. 
En diferentes estudios, hemos analizado el recuerdo de las emociones de futuros profesores de primaria y secundaria sobre distintos contenidos de ciencias, tanto en la etapa de primaria como de secundaria. Estos resultados indican que los profesores en formación tienen un recuerdo emocionalmente muy positivo de las asignaturas de ciencias en primaria cuando ellos fueron alumnos. En el gráfico 1, se refleja el recuerdo de estas emociones en muestras de maestros en formación de las Universidades de Extremadura (UEX), Autónoma de Barcelona (UAB) y Las Palmas de Gran Canaria (ULP) (Brígido et al., 2013b). Destacan emociones positivas como curiosidad, entusiasmo o interés. En cambio, emociones negativas como frustración, odio o ansiedad solo son señaladas por una mínima parte de los encuestados. Un resultado semejante se obtiene con los futuros profesores de secundaria de distintas especialidades durante la realización del Curso de Aptitud Pedagógica (CAP) en el curso 2008-09 en la UEX (Borrachero et al., 2011).

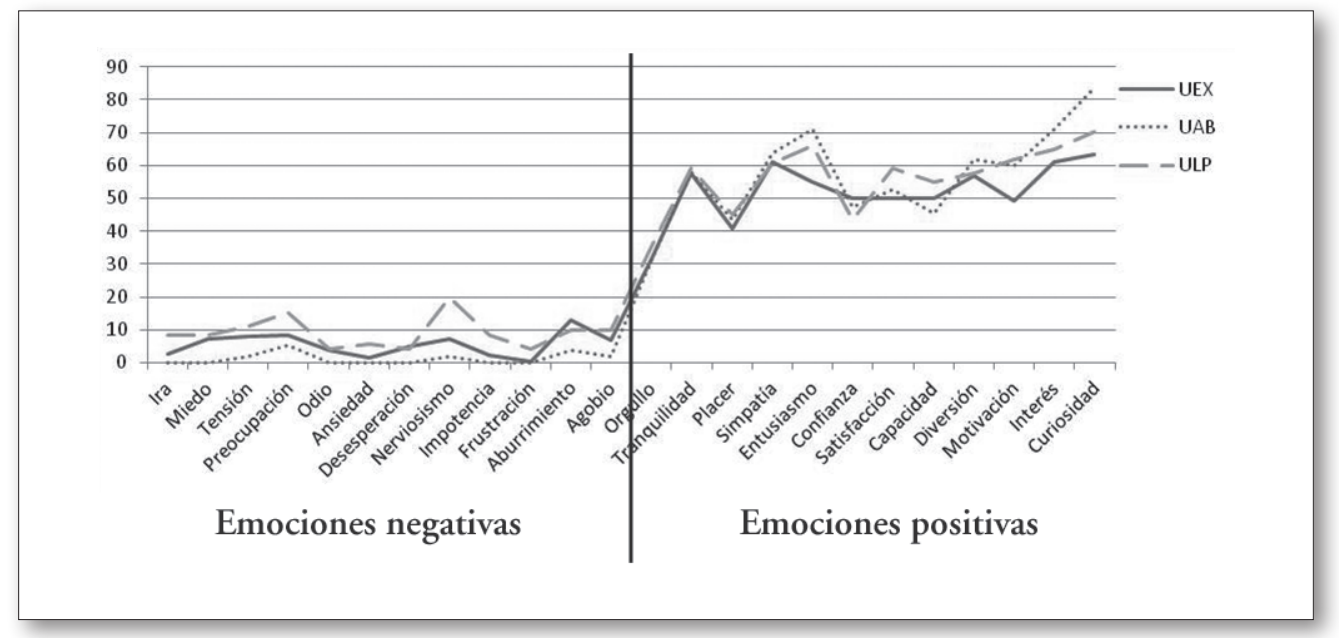

Gráfico 1. Porcentaje de emociones en el aprendizaje de Conocimiento del medio natural en primaria de maestros en formación de tres universidades (Brígido et al., 2013b).

Sin embargo, el recuerdo de su propia etapa de estudiantes de secundaria tiene muchos más matices. En estudios con diferentes muestras de varias universidades existe coincidencia en que el recuerdo de las emociones de futuros profesores de primaria hacia los contenidos de Biología y Geología en secundaria es muy positivo; en cambio, el recuerdo de sus emociones hacia los contenidos de Física y Química en secundaria es mayoritariamente negativo. Como ejemplo mostramos en el gráfico 2, en porcentajes, el recuerdo en su etapa de secundaria de las emociones de maestros en formación de la Universidad de Extremadura hacia el aprendizaje de contenidos de Ciencias naturales (Biología y Geología) y de Física y Química (Brígido et al., 2009, 2010, 2013 a y b). 


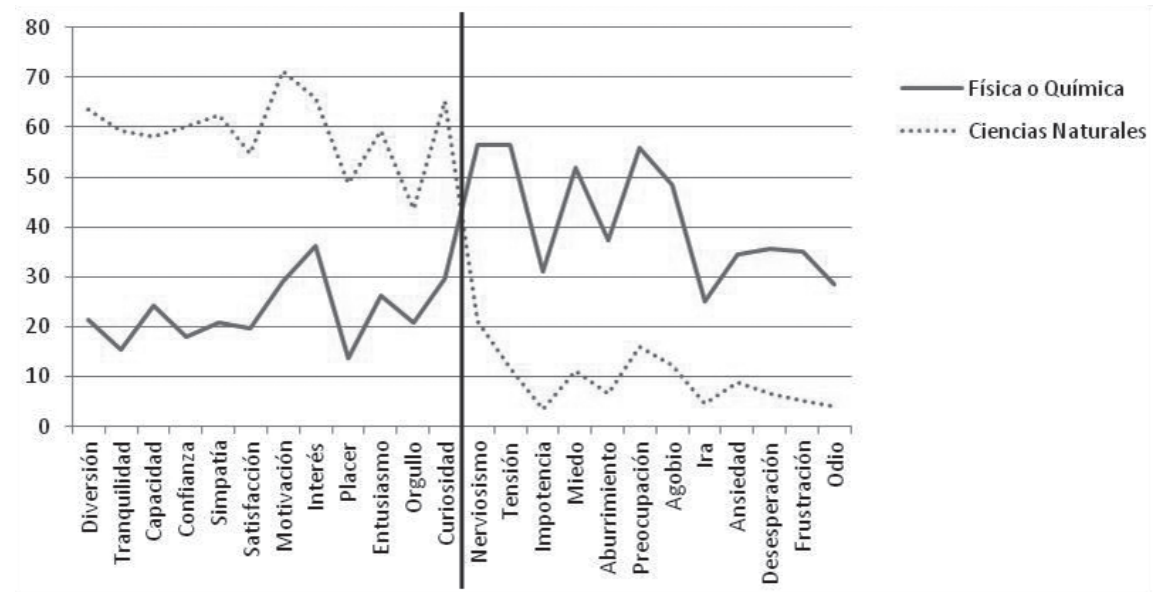

Emociones positivas Emociones negativas

Gráfico 2. Porcentaje del recuerdo de las emociones en secundaria hacia la Física y Química y las Ciencias naturales, en una muestra de maestros en formación de la UEX (Brígido et al., 2009, 2010 y $2013 a$ y b).

En Ciencias naturales la mayoría de las emociones que recuerdan son positivas, como motivación, interés o curiosidad; entre las negativas únicamente destacan, aunque en mucho menor porcentaje, la preocupación y el aburrimiento.

Para los futuros maestros, el recuerdo de las asignaturas de Física y Química en su paso por la secundaria es completamente diferente, pues el recuerdo de estas materias les genera emociones negativas fuertes como miedo, tensión o desesperación; entre las positivas destacan el interés y la curiosidad. Esto va a ser una constante que nos alerta de que en Física y Química tenemos un grave problema en la etapa de secundaria.

Desde la psicología se han estudiado las atribuciones causales como los procesos que permiten a los perceptores atribuir las causas de un efecto emocional determinado (Manassero, 2013). En el grupo de investigación, hemos analizado las causas de estas emociones en secundaria en muestras de estudiantes de primer curso de grado de quince carreras universitarias de distintos campos, y en profesores en formación de primaria y secundaria. Centrándonos en la asignatura de Física, que es la que genera las mayores emociones negativas, observamos en los gráficos 3 y 4 que existen notables diferencias en la atribución de causas entre los futuros profesores de primaria y los de secundaria que se encuentran realizando el Máster Universitario de Formación del Profesorado de Educación Secundaria (MUFPES) en las especialidades de Física y Química, Biología y Geología y Matemáticas de la UEX.

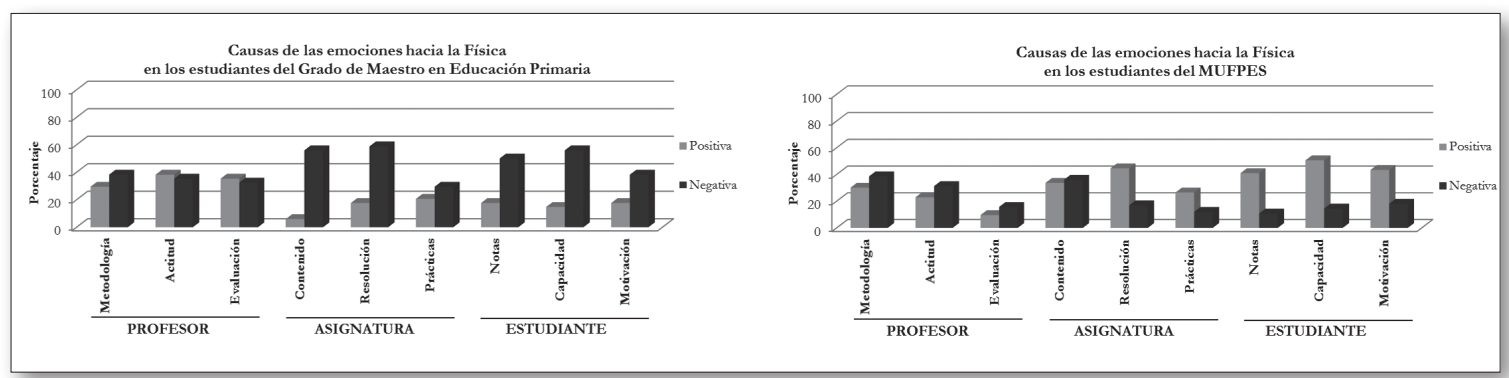

Gráficos 3 y 4. Porcentaje de las causas de las emociones hacia la Física en secundaria en maestros en formación (gráfico 3 ) y en estudiantes del Máster de Secundaria (gráfico 4). 
En los futuros maestros, las atribuciones de causa de las emociones positivas son en mayor porcentaje hacia el profesorado (la metodología de enseñanza, la actitud y el sistema de evaluación) que hacia el contenido de la asignatura (la propia materia, la resolución de problemas y las actividades prácticas) y hacia sí mismos como estudiantes (los resultados académicos, la propia capacidad para aprender y la motivación). Las causas de las emociones negativas las atribuyen sobre todo a sí mismos como estudiantes, al propio contenido de la asignatura de Física y a la resolución de problemas. Para Korthagen (2010), los estudiantes recuerdan a sus buenos profesores fundamentalmente por cualidades emocionales, pero en este caso también les atribuyen la causa de sus emociones positivas hacia la Física.

En cambio, para los futuros profesores de secundaria de las especialidades de Ciencias y Matemáticas las causas de las emociones negativas las atribuyen fundamentalmente al profesorado y al propio contenido de la asignatura de Física, mientras que las causas de las emociones positivas las atribuyen a sí mismos como estudiantes y a las actividades de resolución de problemas y de laboratorio.

Para Manassero (2013), los éxitos que se atribuyen a causas internas originan una mayor autoestima que el éxito atribuido externamente; en cambio, el fracaso atribuido a causas internas origina más baja autoestima que el fracaso atribuido externamente. Los resultados de los futuros profesores de secundaria atribuyen las emociones positivas fundamentalmente a causas internas y las negativas a causas externas. Sin embargo, los resultados de los futuros maestros nos resultan sorprendentes porque las atribuciones de las emociones positivas se asignan a causas externas (el profesorado), mientras que las emociones negativas se asignan al contenido y a causas internas (el propio estudiante).

\section{Las emociones hacia la propia enseñanza de las ciencias de los profesores en formación}

En la formación inicial, las primeras experiencias de enseñanza durante las prácticas de enseñanza son emocionalmente muy fuertes y pueden ser traumáticas para los futuros profesores, que en esta etapa son especialmente vulnerables. Las emociones negativas en las primeras experiencias de enseñanza como ansiedad, inseguridad, irritación o decepción pueden fijar estrategias conductistas, dirigidas hacia el control y la supervivencia, que son muy resistentes al cambio en el futuro (Frenzel et al., 2011). Ritchie et al. (2011), en un estudio con un profesor de ciencias principiante, señalan que las emociones positivas están relacionadas con el logro de las expectativas positivas y con el fracaso de las negativas; en cambio, las emociones negativas están relacionadas con el fracaso de las expectativas positivas.

Para Oosterheert y Vermunt (2001), la regulación emocional es un componente funcional para aprender a enseñar ciencias. La formación inicial del profesorado se constituye como un espacio en el que se deben considerar estos aspectos para que los profesores en formación puedan controlar y mejorar los efectos de sus emociones en la dinámica del aula.

En numerosas muestras de maestros en formación de varias universidades españolas hemos comparado el recuerdo de sus emociones en el aprendizaje de las distintas materias de ciencias, con las que vaticinan que sentirán hacia la enseñanza de estas materias cuando tengan que enseñarlas, y con las que realmente han sentido cuando han tenido que enseñarlas durante las prácticas de enseñanza. En ambos casos, las emociones como docentes están influidas por su propia escolaridad: las emociones como docentes hacia una materia se corresponden con el recuerdo de sus emociones hacia esa misma materia cuando ellos eran alumnos. En el gráfico 5, se muestra, para maestros en formación de la UEX, el recuerdo de sus emociones hacia las Ciencias naturales y las que sienten hacia la enseñanza de esos contenidos después de sus prácticas de enseñanza (Brígido et al., 2010 y 2013a). 


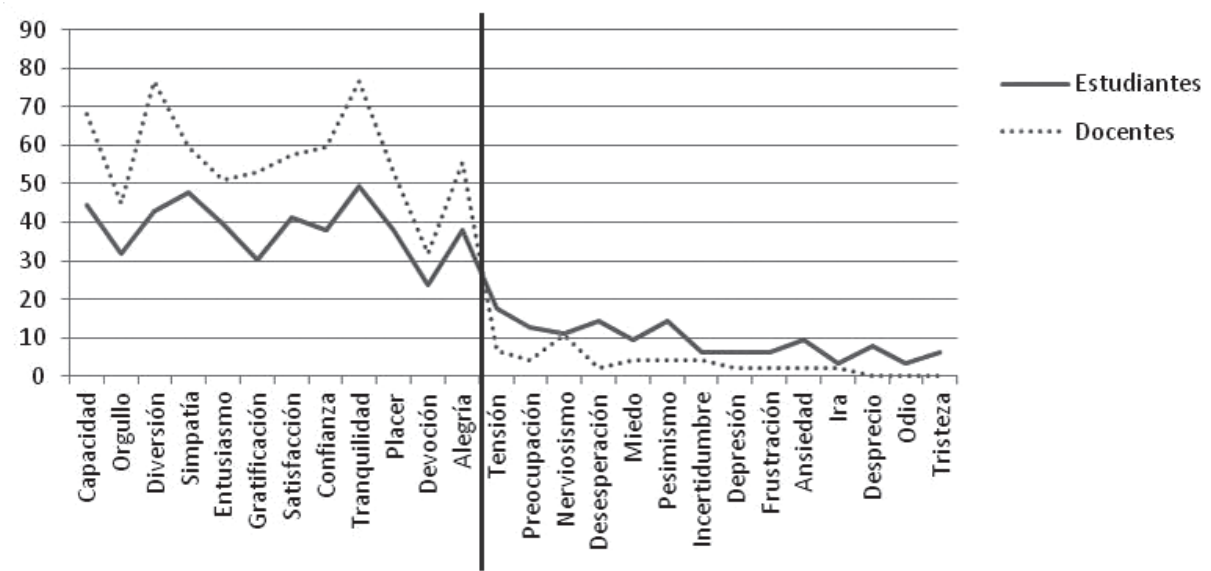

\section{Emociones positivas Emociones negativas}

Gráfico 5. Porcentaje de emociones de maestros en formación hacia las Ciencias naturales, como estudiantes de secundaria y como docentes en prácticas (Brígido et al., 2010 y 2013a).

Se observa la relación entre el recuerdo de las emociones hacia las Ciencias naturales y las que han sentido durante las prácticas, aumentando incluso las emociones positivas y disminuyendo las negativas.

Para Física y Química (gráfico 6) también hay relación, pues disminuyen en general las emociones negativas y mejoran las positivas al impartir estas materias (Brígido et al., 2010 y 2013a), aunque los futuros maestros siguen teniendo emociones muy negativas hacia la docencia de estos contenidos como tensión, miedo, agobio o desesperación. Esto constituye un grave problema pues, si esta situación no mejora, es probable que estos futuros profesores transfieran a sus propios alumnos emociones negativas hacia los contenidos relacionados con la Física y la Química.

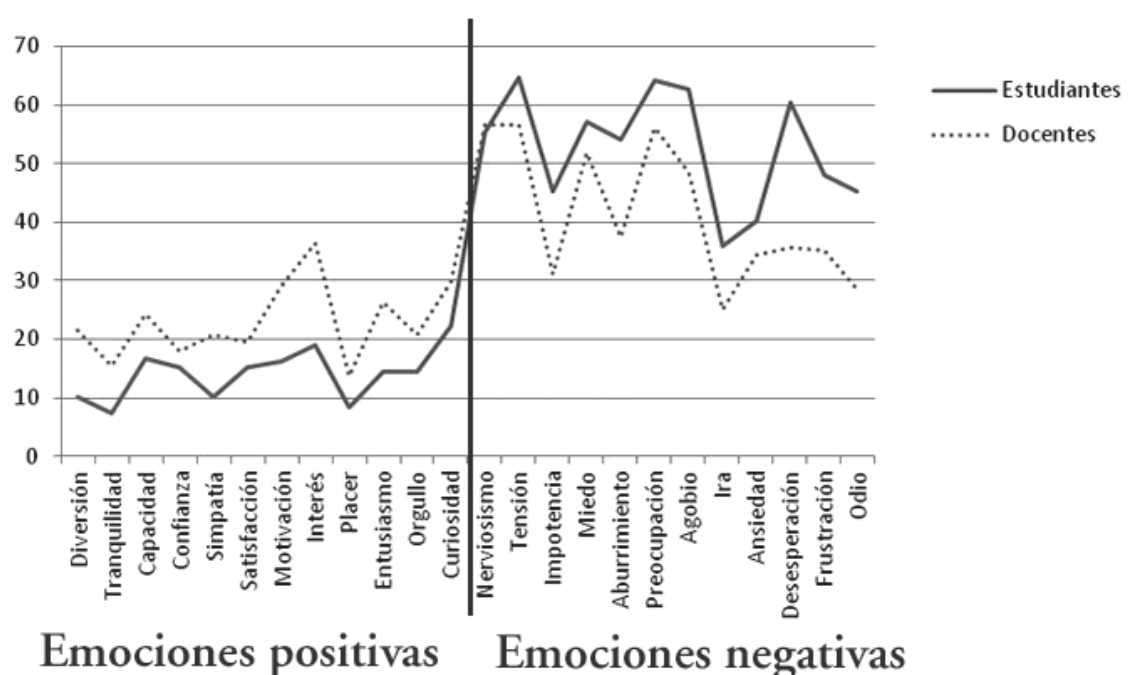

Gráfico 6. Porcentaje de emociones de maestros en formación hacia la Física y Química, como estudiantes de secundaria y como docentes en prácticas (Brígido et al., 2010 y 2013a). 
En profesores de secundaria en formación, hemos realizado estudios con muestras del antiguo Curso de Aptitud Pedagógica (CAP) y del actual Máster Universitario de Formación del Profesorado de Educación Secundaria (MUFPES), ambos cursos de postgrado realizados después del grado o la licenciatura correspondiente (Borrachero et al., 2013a y b, 2014; Costillo et al., 2013a).

En general, los futuros docentes de secundaria muestran emociones positivas hacia las enseńanza de las asignaturas que se corresponden con su especialidad y negativas frente al resto, con dos excepciones: la Biología, en la que todos muestran emociones positivas, y la Física, en la que incluso los futuros profesores de la especialidad de Física y Química muestran casi en la misma medida emociones positivas y negativas. En todos los casos, estas emociones se corresponden con las que sintieron cuando ellos eran estudiantes de estas materias en su etapa de secundaria.

Los resultados tienen muchos más matices cuando se cruzan con otras variables. Para no ser exhaustivos en los datos, mostramos la diferencia por sexos en las emociones hacia la enseñanza de la Biología (gráfico 7) y hacia la enseñanza de la Física (gráfico 8), en una muestra de licenciados en carreras de Ciencias e Ingeniería durante la realización del CAP (Borrachero et al., 2014).

Como hemos reiterado, las emociones son muy positivas en la enseñanza de la Biología, pero las mujeres muestran más intensidad en las emociones, tanto en las positivas como en las negativas (gráfico 7). Este resultado concuerda con los estudios que señalan que las emociones tienen más influencia en las chicas que en los chicos (Hazari et al., 2007).

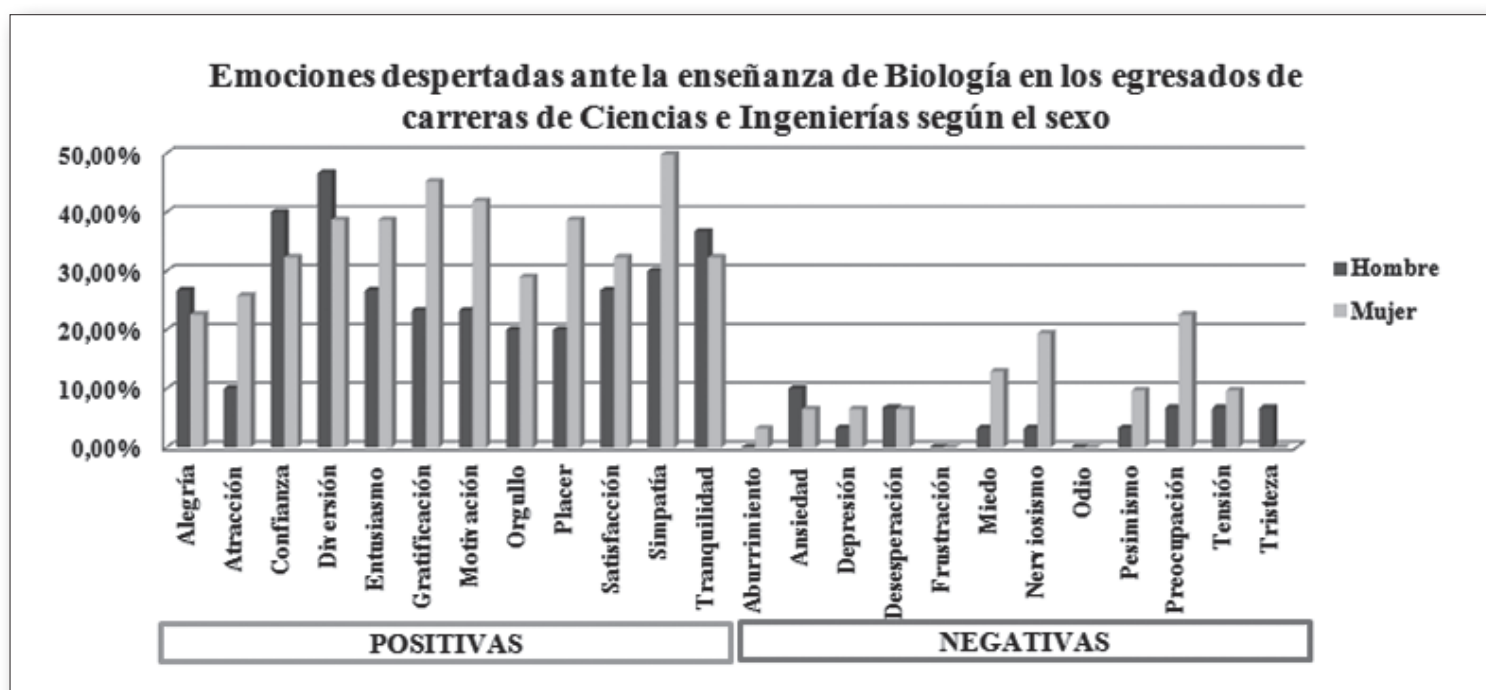

Gráfico 7. Porcentaje de emociones sobre la enseñanza de la Biología de estudiantes del CAP de las especialidades de Ciencias e Ingeniería, diferenciando hombres y mujeres (Borrachero et al., 2014).

En la misma muestra anterior, al preguntar sobre las emociones en la enseńanza de la Física, encontramos que aumentan las emociones negativas y hay una clara diferencia entre hombres y mujeres: las emociones son más positivas en los hombres que en las mujeres en la enseñanza de esta materia (gráfico 8). 


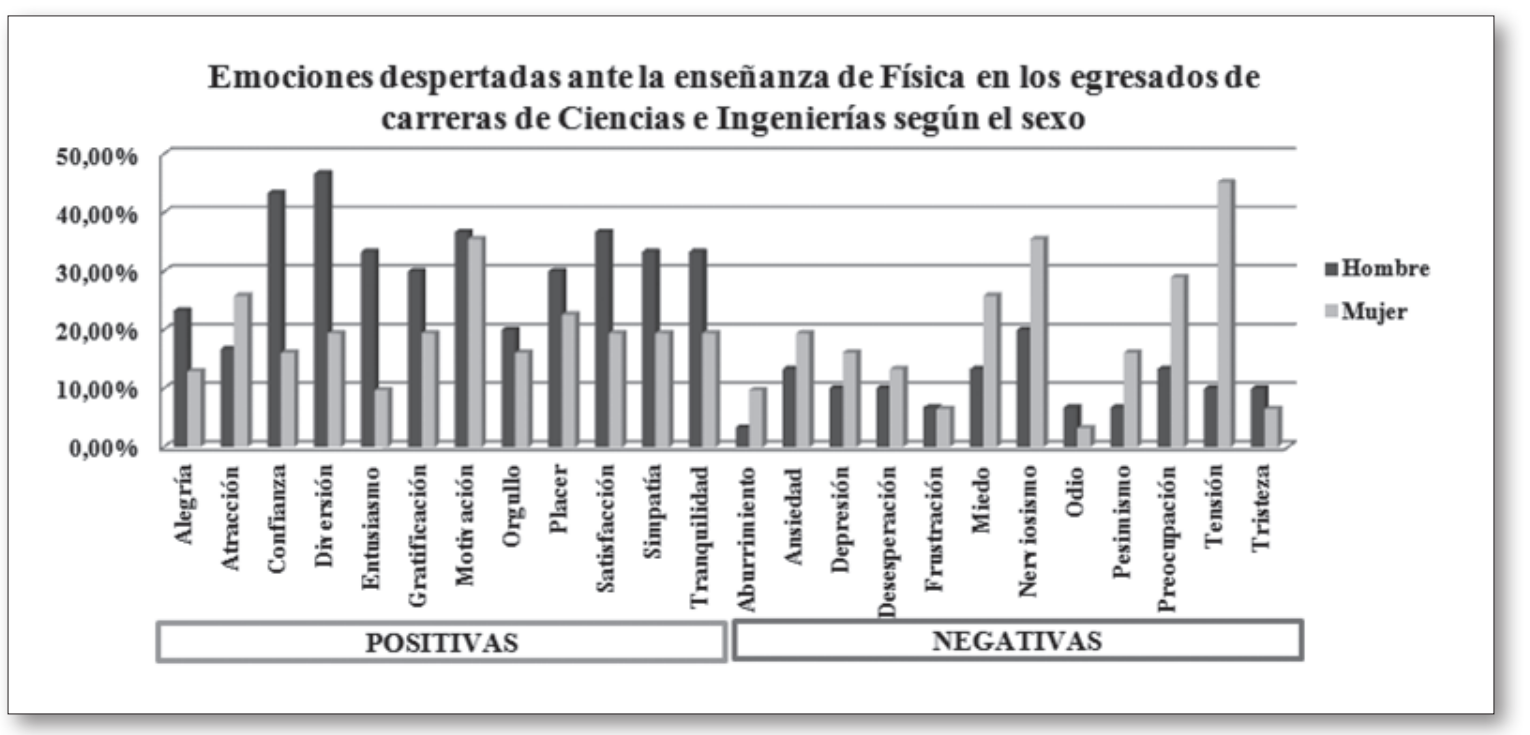

Gráfico 8. Porcentaje de emociones sobre la enseńanza de la Física de estudiantes del CAP de las especialidades de Ciencias e Ingeniería, diferenciando hombres y mujeres (Borrachero et al., 2014).

Inicialmente señalamos la necesidad de tener una mirada amplia y multidisciplinar en la investigación sobre las emociones en la enseñanza-aprendizaje de las ciencias y de tener en cuenta otros muchos constructos. Como ejemplo, mostramos algunos resultados sobre la autoeficacia, que es la creencia en la propia competencia en la realización de una tarea (Bandura, 1986). Si un profesor se considera competente en la enseñanza de una materia, tendrá más motivación y compromiso, considerará los retos como estimulantes e intensificará los logros personales y sus expectativas de éxito. En cambio, los profesores con baja autoeficacia evitarán enseñar los conceptos difíciles o les dedicarán menos tiempo. La autoeficacia está estrechamente relacionada con la autorregulación y es una poderosa variable para predecir la conducta en el aula de los profesores y el logro de los estudiantes (Cakiroglu et al., 2012).

Los resultados con profesores en formación, tanto de primaria (Brígido et al., 2013a) como de secundaria (Borrachero et al., 2013b; Costillo et al., 2013b), indican que para la enseñanza de la Biología la autoeficacia no tiene influencia en sus emociones. En cambio la autoeficacia sí influye en las emociones hacia la enseñanza de materias en las que las emociones son más negativas.

En el gráfico 9, se observa cómo para los maestros en formación que tienen una alta autoeficacia hacia la enseńanza de la Física y la Química aumentan las emociones positivas y disminuyen las negativas hacia la enseñanza de estas materias, con respecto a los que tienen baja autoeficacia (Brígido et al., 2013a). 


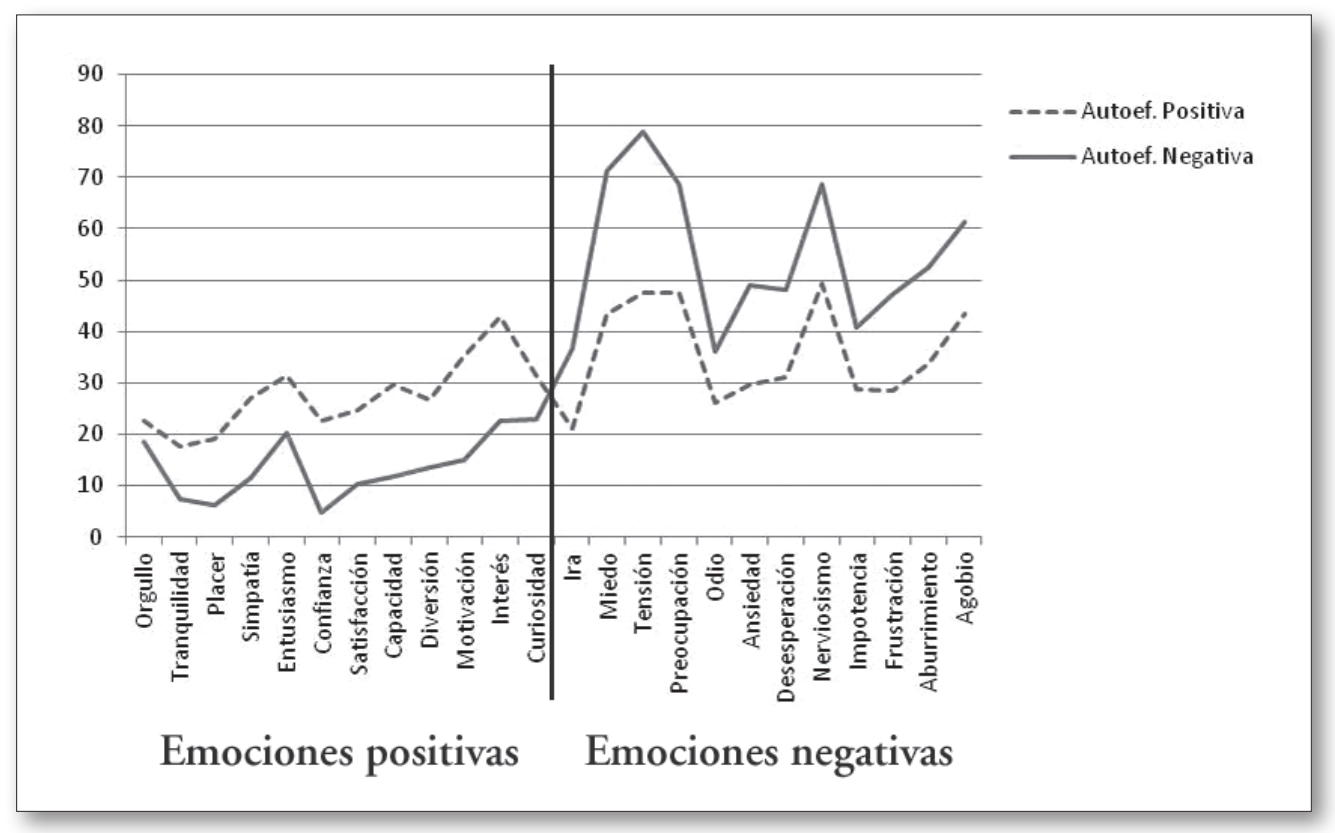

Gráfico 9. Porcentaje de emociones en la enseńanza de la Física o Química de maestros en formación con autoeficacia positiva y negativa (Brígido et al., 2013a).

También para los profesores de secundaria en formación (Borrachero et al., 2013a; Costillo et al., 2013), la autoeficacia influye en el caso de las materias que generan más emociones negativas. En el gráfico 10 se observa, para una muestra de estudiantes del CAP de distintas especialidades, cómo las emociones positivas hacia la enseńanza de la Física aumentan y las negativas disminuyen para los profesores que tienen mayor autoeficacia hacia la enseñanza de esta materia.

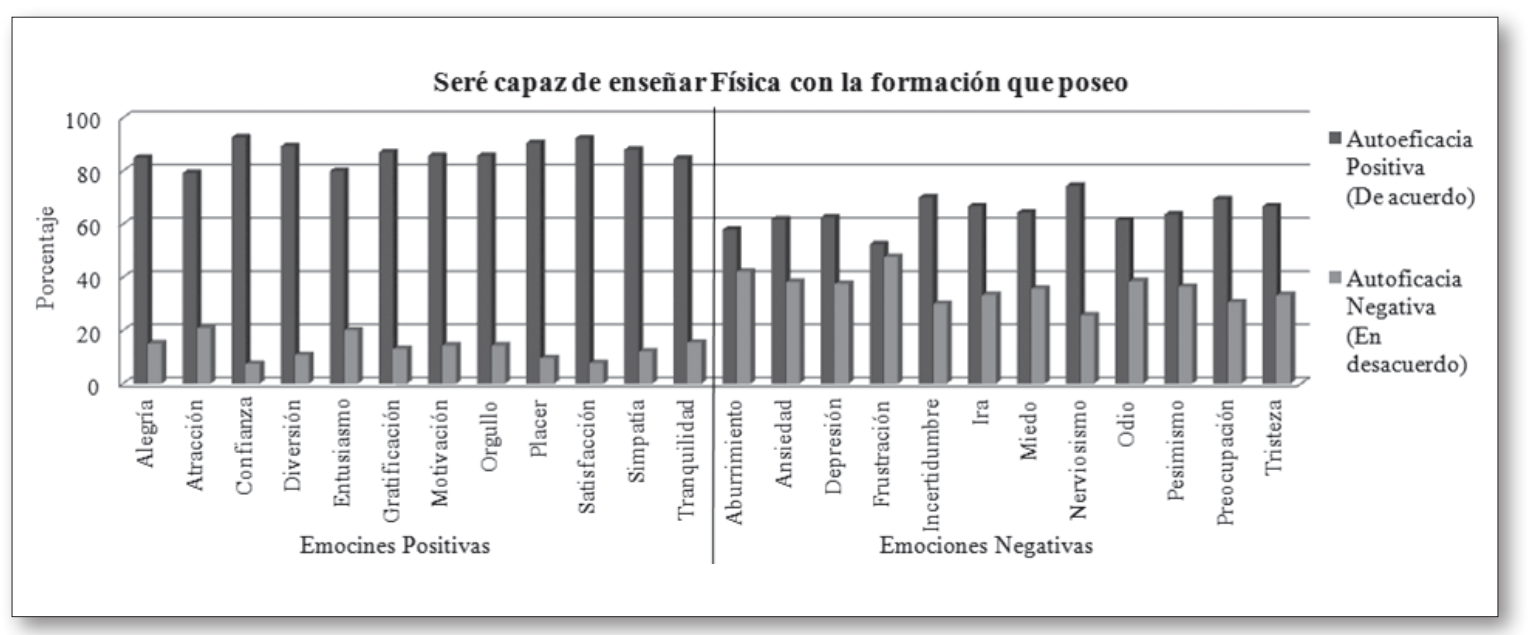

Gráfico 10. Porcentaje de emociones y autoeficacia hacia la enseñanza de la Física en profesores de secundaria en formación de distintas especialidades (Borrachero et al., 2013a).

Estos resultados nos indican una línea de trabajo en la que incidir para mejorar la autoeficacia y las emociones hacia la Física y la Química durante la formación inicial del profesorado. 


\section{Las emociones en el desarrollo profesional del profesorado de ciencias}

El desarrollo profesional tiene que ir unido al personal y al social teniendo en cuenta los aspectos afectivos, fomentando la colaboración constructiva, fortaleciendo la cultura de los centros y construyendo sobre las buenas prácticas que los profesores estén ya realizando (Hargreaves, 1996; Mellado, 2003). Como señala metafóricamente Day (1999), el cambio del profesorado es un asunto de la cabeza pero también del corazón, y difícilmente se realizan cambios didácticos si estos no compensan afectivamente y contribuyen a dar más satisfacción personal en el propio trabajo.

En una investigación longitudinal basada en la reflexión orientada, llevada a cabo durante cinco años con una profesora de Física de secundaria con 25 años de experiencia docente (Peme et al., 2008), la profesora mostró el primer ańo de la investigación un modelo mayoritariamente centrado en los alumnos (un 57\% de acciones). Sin embargo, durante el segundo y tercer año de la investigación, las acciones centradas en los estudiantes disminuyeron (un 52 y un $41 \%$ respectivamente) y aumentaron las centradas en la explicación de la profesora y en los contenidos. Esto no se debió a razones cognitivas, ya que la profesora estaba mejor preparada para implementar estrategias innovadoras, sino a la angustia que le causaba implementar estas estrategias en un centro de naturaleza conservadora. Solo el cuarto y quinto año de la investigación las acciones centradas en los alumnos aumentaron (un 70 y $72 \%$ respectivamente), a medida que fue cobrando seguridad en su propio modelo y, con la ayuda de la orientadora, pudo llevar al aula las estrategias planificadas.

La investigación anterior nos indica la dificultad de que se realicen cambios didácticos, y sobre todo que se consoliden, de una forma individual y a contracorriente de la "cultura" educativa del centro y de las formas socialmente asumidas. Los obstáculos al cambio del profesorado son en muchos casos de naturaleza emocional y social (Bell y Gilbert, 1994). Cross y Hong (2011), después de investigar el cambio de numerosos profesores de ciencias, consideran que los profesores filtran las reformas para ver si coinciden con sus metas sobre la enseńanza. Esta coincidencia o discrepancia les genera emociones positivas o negativas, que influyen en su identidad y en sus roles y condicionan la puesta en práctica de las reformas. Pero aun cuando las reformas coincidan con sus metas, la puesta en práctica de estas también puede generarles emociones negativas como ansiedad, inseguridad, ira, o frustración debido a múltiples causas como la falta de tiempo, la falta de recursos y materiales adaptados (Pro et al., 2008), o por no sentirse comprendidos y apoyados por los propios compañeros y directivos, lo que puede tener como consecuencia el rechazo a reformas innovadoras. Este tipo de obstáculos ha incidido en que algunos aspectos innovadores incluidos en algunas de las reformas en España, como las competencias o los contenidos procedimentales en las asignaturas de ciencias, sobre los que en gran parte se basa la evaluación del Informe PISA para los estudiantes de ESO, apenas se hayan puesto en práctica en las aulas de secundaria (Banet, 2007; Pro, 2006).

\section{LA INTERVENCIÓN SOBRE LAS EMOCIONES DEL PROFESORADO DE CIENCIAS}

Los resultados anteriores nos aportan datos para el diagnóstico de las emociones en la enseñanzaaprendizaje de las ciencias, pero esto es solo la primera parte. Hay que pasar del diagnóstico a la intervención y hacer que los futuros profesores de ciencias sean emocionalmente competentes, y que sepan diagnosticar y autorregular sus propias emociones a través de programas de intervención que incluyan tanto lo cognitivo como lo afectivo (Bisquerra y Pérez, 2007).

Damasio (2010) señalaba que lo que acarrea emociones negativas solo puede ser contrarrestado generando emociones positivas aún más fuertes. Esto significa que es necesario provocar emociones positivas hacia el proceso de enseñanza-aprendizaje de las ciencias que contrarresten las emociones 
negativas que pudieran haberse generado a lo largo de la escolaridad. Pero no hay que hacerlo en abstracto, al margen de los contenidos y de la actividad científica, sino con actividades y temas de ciencias.

Izquierdo (2013), con el sugestivo título de ¿La química emociona?, considera que hacer ciencia emociona porque es la expresión del deseo de conocer y de comprender el mundo en el que vivimos. Al hacer ciencia, los científicos utilizan un lenguaje interpretativo y emocional que tiende a persuadir y a convencer (Sutton, 1997). Coincidimos con Izquierdo (2013) en que aprender ciencias también debería emocionar; no solo por lo que supone de sorpresa, curiosidad, gratificación, confianza, y otras muchas emociones positivas, sino también por la capacidad de usar el lenguaje como instrumento para la abstracción, la comunicación y la argumentación.

Sin embargo, en la escuela a menudo se ha abusado de la transmisión de la ciencia como un conjunto de verdades acabadas e indiscutibles, alejadas no solo de las inquietudes de los alumnos, sino también de la emoción que supuso en su momento histórico la construcción del conocimiento. Esta forma de plantear el aprendizaje científico genera en muchos alumnos actitudes y emociones negativas hacia determinadas materias, que se transfieren posteriormente a las que tienen como profesores cuando les toca enseñarlas. Este círculo vicioso tiene que romperse fundamentalmente durante la formación inicial del profesorado, proponiendo actividades científicas creativas y emocionantes que combinen situaciones problemáticas abiertas sobre trabajos prácticos. Disponemos ahora de conocimientos de didáctica de las ciencias que nos ofrecen fundamentos teóricos y prácticos para avanzar con una cierta seguridad en este planteamiento.

El programa de intervención que estamos llevando a cabo en la Universidad de Extremadura lo realizamos en equipos interdisciplinares, combinando distintos marcos teóricos y analizando distintas variables. En el programa se realizan tanto actividades generales de afrontamiento y control emocional, como específicas de enseñanza-aprendizaje de las ciencias que permiten hacer un seguimiento cognitivo y emocional. Por ejemplo, una de las actividades propuestas, para realizar en pequeńos grupos, es averiguar las variables de las que depende el período de oscilación del péndulo simple. En ella se combina un problema abierto con actividades prácticas, guiando el desarrollo de procesos propios de la metodología científica a la vez que se analizan las emociones que ello van generando cuando, por ejemplo, se enfrentan a un problema abierto (Thomas y Ritchie, 2012). En cada proceso científico hay una mezcla de emociones positivas y negativas: destacamos, después de las dudas iniciales, la fase de experimentación y representación de los datos, que genera más emociones positivas, algunas tan interesantes como la curiosidad o la confianza. La fase que genera más emociones negativas es la de qué hacer con los datos, qué regularidades existen, qué leyes pueden inferirse de ellos. Pero cuando son capaces de resolverlo y de aplicarlo a problemas prácticos, esto les genera un aumento de la confianza y de la autoestima y emociones positivas como orgullo, alegría y gratificación.

En maestros en formación hemos medido las emociones que sienten hacia el aprendizaje de las ciencias, antes y después de realizar distintas actividades en el laboratorio, y los resultados son esperanzadores. En el gráfico 11, se muestra la evolución de estas emociones, medidas en una escala de frecuencia tipo Likert, en la que 0 es la mínima frecuencia y 3 la máxima. 


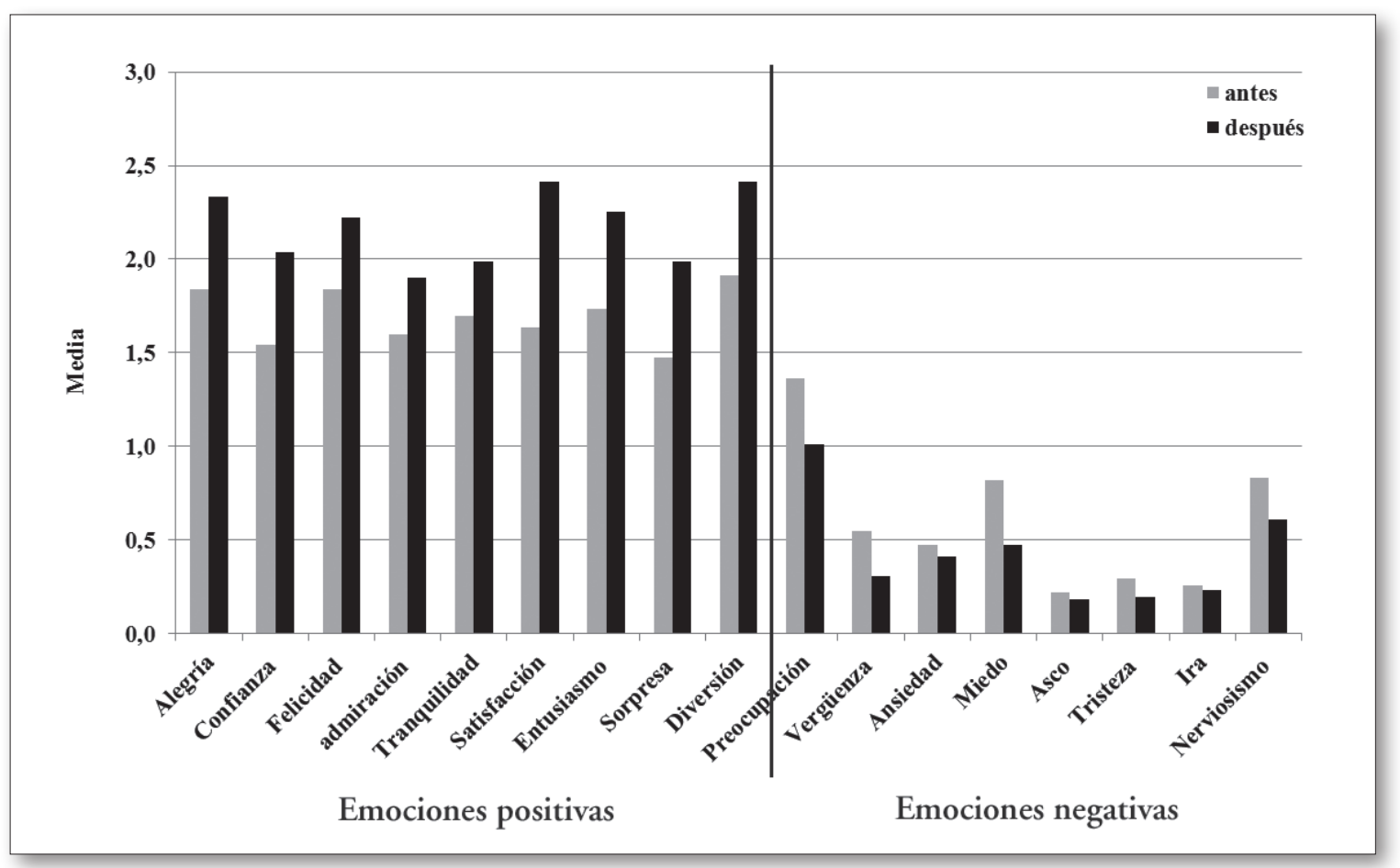

Gráficos 11. Evolución de las emociones de maestros en formación, antes y después de la realización de actividades de ciencias.

Sin embargo, tener un buen conocimiento de la materia y haber sentido emociones positivas hacia su aprendizaje es una condición necesaria, pero no suficiente, para que el profesor aprenda a enseńarla, ya que este conocimiento proposicional no se transfiere directamente a la práctica de la enseńanza. El conocimiento didáctico del contenido se genera y evoluciona a partir de los propios conocimientos académicos (del contenido, de didáctica de las ciencias, de psicopedagogía general, etcétera.), filtrados por las concepciones, los valores, las actitudes y emociones, pero requiere de la implicación y reflexión personal sobre el proceso de enseñanza de la materia específica en contextos escolares concretos. Es necesario planificar actividades de enseñanza creativas y emocionantes que los profesores en formación puedan desarrollar. La reflexión en y sobre la práctica de la enseńanza es lo que permite al profesor en formación analizar y reconstruir sus teorías personales y su modelo de enseñanza, así como generar más conocimiento procedimental y más esquemas prácticos de acción en el aula. El análisis de las metáforas personales es una herramienta que facilita a los futuros profesores la reflexión sobre sus concepciones, modelos personales de enseñanza, emociones y roles.

\section{Las metáforas en la formación del profesorado de ciencias}

Una metáfora es la sustitución o transposición de una idea o concepto por otro que tenga con él una cierta relación de semejanza. Las metáforas no son solo un recurso expresivo, sino que constituyen un mecanismo esencial de la mente que estructura gran parte del sistema conceptual por medio de relaciones metafóricas, y son un principio fundamental del pensamiento y la acción (Lakoff y Johnson, 1986).

Las metáforas personales de los profesores se forman de las propias experiencias como escolares y de las propias experiencias de enseńanza; permiten expresar de forma global las concepciones y el conocimiento práctico; influyen en la conducta en el aula, y son un puente entre lo cognitivo y lo 
afectivo (Zembylas, 2004). En un estudio con futuros profesores de ciencias de secundaria del MUFPES de la UEX (Mellado et al., 2012 y 2013), adaptamos las cuatro categorías de Leavy et al. (2007): la conductista-transmisiva (el alumno como un aprendiz pasivo y el profesor como un transmisor de información), que tiene además la característica de ser una clase centrada en el profesor y el contenido; la cognitiva-constructivista (el alumno como un agente activo en el proceso de construcción de su propio conocimiento y el profesor como un facilitador del proceso); la situada o de aprendizaje social (con similares características que la anterior, pero haciendo más hincapié en el aprendizaje social que en el individual, y están ambas centradas en el alumno y el aprendizaje), y la autorrefenciada, que son metáforas de difícil clasificación y que tienen un componente egocéntrico, cuyo significado es necesario analizar en cada caso.

El mayor número de metáforas personales globales se encuentra dentro de la categoría conductista/ transmisiva (gráfico 12), en la que la metáfora dominante es la del profesor como trasmisor de conocimientos, habilidades y destrezas, y la del alumno es la de receptor pasivo de estos conocimientos (por ejemplo, sargento instructor, gasolinera, juez, pavo real, etcétera). Si como señalan numerosas investigaciones (Tobin et al., 1994), los profesores en formación toman como referencia los modelos de enseñanza en los que ellos mismos fueron enseñados, el gran número de metáforas conductistas/transmisivas expresadas por los futuros profesores de secundaria puede ser un reflejo del tipo de enseñanza que ellos mismos recibieron. Un porcentaje significativo de metáforas son cognitivas/ constructivistas (por ejemplo, equilibrio entre reactivos, pastelera, cemento, director de teatro, etcétera), en las que el profesor se considera un educador y facilitador, y el alumno un aprendiz activo que construye poco a poco un aprendizaje significativo. Otro resultado es el pequeño porcentaje de metáforas situadas (por ejemplo, pastor, barco que guia, luz que alumbra el camino, etcétera), que son metáforas identificadas como iluminación, viaje, trayecto, descubrimiento, exploración, búsqueda, guía, etcétera. Entre las metáforas autorreferenciadas, hay muchos ejemplos de animales (por ejempo, avestruz, hormiga más que cigarra, tortuga, lince, etcétera). A diferencia de las metáforas totales, en las que el mayor número pertenecían a la categoría conductista/transmisiva, en las emocionales hay un gran porcentaje de metáforas autorreferenciadas y cognitivas/constructivistas (gráfico 13).

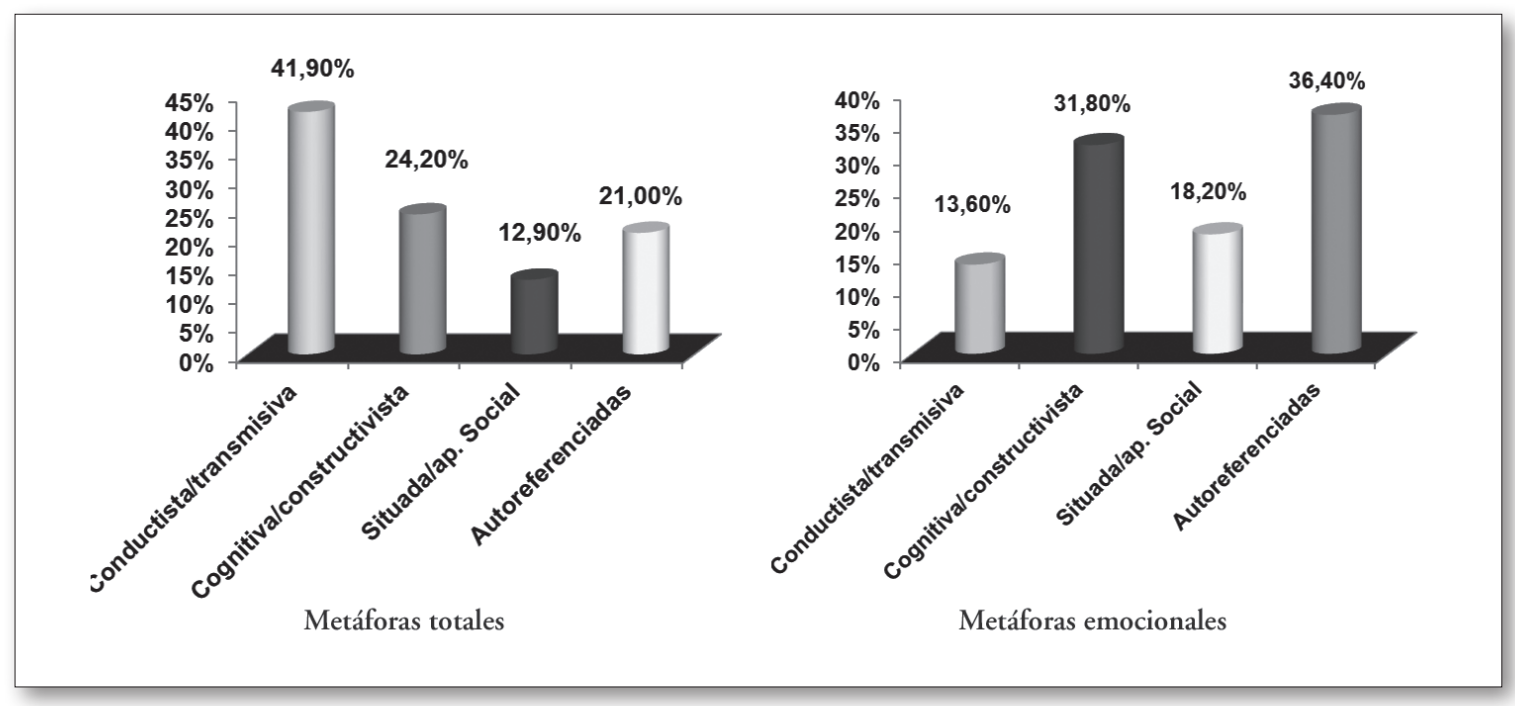

Gráficos 12 y 13. Porcentaje de metáforas totales y emocionales de futuros profesores de ciencias de secundaria (Mellado et al., 2012 y 2013). 
El análisis de las metáforas y, en particular, de las emocionales durante las prácticas de enseñanza, un período decisivo en el que se consolidan rutinas de enseñanza y en el que los futuros profesores están sometidos a una fuerte presión emocional, puede ser un recurso más que contribuya a la formación cognitiva y emocional de estos profesores.

\section{REFLEXIÓN FINAL}

Después de años de olvido, las emociones comienzan a formar parte de la agenda de investigación en didáctica de las ciencias. Sin embargo, nuestra intención al estudiar las emociones no es sustituir el reduccionismo racionalista por el emocional, sino que emociones y razón se complementen. Como dice Antonio Damasio (2010), se trata de establecer un dialogo entre la razón y las emociones y de entender cómo se relacionan.

El estudio de las emociones desde la didáctica de las ciencias puede aportar datos para que tanto el alumnado como el profesorado sean conscientes de la importancia de sus emociones, de que pueden ser vulnerables emocionalmente, así como para establecer programas de intervención metacognitivos y metaemocionales, tanto en el aprendizaje como en la formación del profesorado, para que alumnos y profesores puedan conocer sus emociones, controlarlas y autorregularlas.

Las emociones negativas son en muchas ocasiones un obstáculo para el proceso de enseñanzaaprendizaje de las ciencias. El reto es, a través de actividades científicas creativas y emocionantes, conseguir sustituir las emociones negativas por el placer, el orgullo, la satisfacción, la alegría, la confianza y tantas otras emociones positivas que a lo largo de la historia han hecho de la actividad científica una aventura del pensamiento y una empresa profundamente humana, que ha contribuido decisivamente a la comprensión de la naturaleza y al progreso de la humanidad.

\section{AGRADECIMIENTOS:}

Este trabajo ha sido financiado por los Proyectos de Investigación EDU2009-12864 del Ministerio de Ciencia e Innovación y EDU2012-34140 del Ministerio de Economía y Competitividad, y por la Junta de Extremadura.

\section{REFERENCIAS BIBLIOGRÁFICAS}

Abrahams, I. (2009). Does Practical Work Really Motivate? A study of the affective value of practical work in secondary school science. International Journal of Science Education, 31(17), pp. 23352353.

Acevedo, J.A. (2009). Conocimiento didáctico del contenido para la enseñanza de la naturaleza de la ciencia (I): el marco teórico. Revista Eureka sobre Enseñanza y Divulgación de las Ciencias, 6(1), pp. 21-46.

Adúriz-Bravo, A. e Izquierdo, M. (2002). Acerca de la didáctica de las ciencias como disciplina autónoma. Revista Electrónica de Enseñanza de las Ciencias, 1(3), pp. 130-140.

Angie, A.D.; Connelly, S.; Waples, E.P. y Vykinta Kligyte, V. (2011). The influence of discrete emotions on judgement and decision-making: A meta-analytic review. Cognition and Emotion, 25 (8), pp. 1393-1422. http://dx.doi.org/10

Bandura, A. (1986). Social foundations of thought and action: A social cognitive theory. New Jersey: Prentice-Hall. 
Banet, E. (2007). Finalidades de la educación científica en secundaria: opinión del profesorado sobre la situación actual. Enseñanza de las Ciencias, 25(1), pp. 5-20.

Bell, B. y Gilbert, J. (1994). Teacher development as professional, personal and social development. Teaching and Teacher Education, 10(5), pp. 483-497. http://dx.doi.org/10.1016/0742-051X(94)90002-7

Bellocchi, A.; Ritchie, S.M.; Tobin, K.; Sandhu, M. y Sandhu, S. (2013). Exploring emotional climate in preservice science teacher education. Cultural Studies of Science Education, 8(3), pp. 529-552.

http://dx.doi.org/10.1007/s11422-013-9526-3

Bisquerra, R. (2000). Educación emocional y bienestar. Barcelona: Praxis.

Bisquerra, R. y Pérez, N. (2007). Las competencias emocionales. Educación XXI, 10, pp. 61-82.

Borrachero, A.B.; Brígido, M.; Bermejo, M.L.; Gómez Del Amo, R. y Mellado, V. (2011). Las emociones de los futuros profesores de secundaria sobre el aprendizaje y la enseñanza de las ciencias. International Journal of Developmental and Educational Psychology, 3(1), pp. 521-530.

Borrachero, A.B.; Brígido, M.; Costillo, E.; Bermejo, M.L. y Mellado, V. (2013b). Relationship between Self-Efficacy Beliefs and Emotions of Future Teachers of Physics in Secondary Education. Asia-Pacific Forum on Learning and Teaching Science, 14(2), Art. 5.

Borrachero, A.B.; Brígido, M.; Mellado, L.; Costillo. E. y Mellado, V. (2014). Emotions in prospective secondary teachers when teaching science content, distinguishing by gender. Research in Science \& Technological Education, 32(2), pp. 182-215.

Borrachero, A.B.; Costillo, E. y Melo, L.V. (2013a). Diferencias en las emociones como estudiante y docente de asignaturas de ciencias de secundaria. En V. Mellado, L.J. Blanco, A.B. Borrachero y J.A. Cárdenas (Eds.). Las Emociones en la Enseñanza y el Aprendizaje de las Ciencias y las Matemáticas. Badajoz: DEPROFE, pp. 373-394.

http://dx.doi.org/10.1080/02635143.2014.909800

Brígido M.; Bermejo, M.L.; Conde, M.C. y Mellado, V. (2010). The emotions in teaching and learning Nature Sciences and Physics/Chemistry in pre-service primary teachers. US-China Education Review, 7(12), pp. 25-32.

Brígido, M.; Borrachero, A.B.; Bermejo, M.L. y Mellado, V. (2013a). Prospective primary teachers' self-efficacy and emotions in science teaching. European Journal of Teacher Education, 36(2), pp. 200-217.

http://dx.doi.org/10.1080/02619768.2012.686993

Brígido, M.; Caballero, A.; Bermejo, M.L.; Conde, M.C. y Mellado, V. (2009). Las emociones en ciencias de Maestros de Educación Primaria en Prácticas. Campo Abierto, 28(2), pp. 153-177.

Brígido, M.; Couso, D.; Gutiérrez, C. y Mellado, V. (2013b). The Emotions about Teaching and Learning Science: A Study of Prospective Primary Teachers in Three Spanish Universities. Journal of Baltic Science Education, 12(3), pp. 299-311.

Cakiroglu, J.; Capa-Aydin, Y. y Woolfolk Hoy, A. (2012). Science Teaching Efficacy Beliefs, en B.J. Fraser, K. Tobin y C. McRobbie (Eds.). Second International Handbook of Science Education (Vol. 1). The Netherlands: Springer, pp. 449-462.

Camps, V. (2012). El gobierno de las emociones. Barcelona: Herder.

Costillo. E.; Borrachero, A.B.; Brígido, M. y Mellado, V. (2013a). Las emociones sobre la enseñanza-aprendizaje de las ciencias y las matemáticas de futuros profesores de Secundaria. Revista EUREKA de Enseñanza y Divulgación de las Ciencias, 10(n. ${ }^{\circ}$ extra), pp. 514-532.

Costillo, E.; Cubero, J. y CAÑAda, F. (2013b). Emociones y autoeficacia de profesores de secundaria en formación ante la enseñanza y el aprendizaje de las ciencias. En V. Mellado, L.J. Blanco, A.B. 
Borrachero y J.A. Cárdenas (Eds.). Las emociones en la enseñanza y el aprendizaje de las ciencias y las matemáticas (Vol. II). Badajoz: UEX-DEPROFE, pp. 395-415.

Cross, D.I. y Hong, J.Y. (2011). Beliefs and professional identity: Critical construct in examining yhe impact of reform on the emotional experiences of teachers. En P.A. Schtz y M. Zembylas (eds.): Advances in teacher emotion research. Dordrecht: Springer, pp. 273-296.

Day, CH. (1999). Developing teachers, the challenges of lifelong learning. London: Falmer Press.

DíAz, J.L. y Flores, E.O. (2001). La estructura de la emoción humana: un modelo cromático del sistema afectivo. Salud Mental, 24(4), pp. 20-35.

Damasio, A. (1996). El error de Descartes. Barcelona: Crítica.

Damasio, A. (2005). En busca de Spinoza. Barcelona: Crítica.

Damasio, A. (2010). Y el cerebro creó al hombre. Barcelona: Destino

Darwin, CH. (1872). The expression of the emotions in man and animals. London: John Murray, Albemarle Street. [Versión española de (2009). La expresión de las emociones. Pamplona: LAETOLI]. http://dx.doi.org/10.1037/10001-000

Dolan, R.J. (2002). Emotion, Cognition, and Behavior, Science, 298, pp. 1191-1194.

Dos Santos, F.M.T y Mortimer, E.F. (2003). How emotions shape the relationship between a chemistry teacher and her high school students. International Journal of Science Education, 25(9), pp. 1095-1110.

http://dx.doi.org/10.1080/0950069032000052216

Fernández-Abascal, E.; Martín, M. y Domínguez, J. (2001). Procesos psicológicos. Madrid: Ediciones Pirámide.

Frenzel, A.C.; Goetz, T.; Stephens, E.J. y Jасов, B. (2011). Antecedents and effects of teachers' emotional experiences: An integrated perspective and empirical test. En P.A. Schutz y M. Zembylas (Eds.). Advances in teacher emotion research. Dordrecht: Springer, pp. 129-151.

Friedrichsen, P.J.; Abell, S.K.; Pareja, E.M.; Brown, P.L.; Lankford, D.M. y Volkmann, M.J. (2009). Does Teaching Experience Matter? Examining Biology Teachers' Prior Knowledge for Teaching in an Alternative Certification Program. Journal of Research in Science Teaching, 46(4), pp. 357-383.

http://dx.doi.org/10.1002/tea.20283

Gardner, H. (2005). Inteligencias múltiples. La teoría en la práctica. Madrid: Paidós.

Garritz, A. (2010). Pedagogical Content Knowledge and the affective domain of Scholarship of Teaching and Learning, International Journal for the Scholarship of Teaching and Learning, 4(2), pp. 1-6.

Garritz, A. y Ortega-Villar, N.A. (2013). El aspecto afectivo en la enseñanza universitaria. Cómo cinco profesores enseñan el enlace químico en la materia condensada. En V. Mellado, L.J. Blanco, A.B. Borrachero y J.A. Cárdenas (Eds.). Las emociones en la enseñanza y el aprendizaje de las ciencias y las matemáticas (Vol. II). Badajoz: UEX-DEPROFE, pp. 277-304.

Gil, D.; Carrascosa, J. y Mártínez, F. (2000). Una disciplina emergente y un campo específico de investigación. En F.J. Perales y P. Cañal (Eds.). Didáctica de las Ciencias Experimentales. Alcoy: Marfil, pp. 11-34.

Goleman, D. (1996). Inteligencia emocional. Barcelona: Kairós.

Gutiérrez, P. y Luengo, M.R. (2003). Orientación vocacional y género. Campo Abierto 23(1), pp. 85-98.

Hargreaves, A. (1996). Profesorado, cultura y modernidad. Madrid: Morata.

Hargreaves, A. (1998). The emotions of teaching and educational change. En A. Hargreaves, A. Lieberman, M. Fullan y D. Hopkins (Eds.). International handbook of educational change. Dordrecht/ Boston/ London: Kluwer Academic Publishers, pp. 558-575. 
Hazari, Z.; TAI, R.H. y SAdler, P.M. (2007). Gender Differences in Introductory University Physics Performance: The Influence of High School Physics Preparation and Affective Factors. Science Education, 91(6), pp. 847-876.

http://dx.doi.org/10.1002/sce.20223

Hernández, P. (2002). Los moldes de la mente. Más allá de la inteligencia emocional. Tenerife: Tafor.

Hugo, D.; Sanmartí, N. y Aduriz-Bravo, A. (2013). Estilos de trabajo emocional del futuro profesorado de ciencias durante el Practicum. Enseñanza de las ciencias, 31(1), pp. 151-168.

IzQuierdo, M. (2013). La química ¡emociona? En V. Mellado, L.J. Blanco, A.B. Borrachero y J.A. Cárdenas (Eds.). Las Emociones en la Enseñanza y el Aprendizaje de las Ciencias y las Matemáticas. Badajoz: DEPROFE, pp. 307-328.

KInd, V. (2009). Pedagogical content knowledge in science education: perspectives and potential for progress. Studies in Science Education, 45(2), pp. 169-204.

http://dx.doi.org/10.1080/03057260903142285

Koballa, T.R. y Glynn, S.M. (2007). Attitudinal and Motivational constructs in science learning. En S.K. Abell y N.G. Lederman (Eds.). Handbook of Research on Science Education. Mahwah, NJ, USA: Erlbaum, pp. 75-102.

Korthagen, F.A. (2010). La práctica, la teoría y la persona en la formación del profesorado. La práctica, la teoría y la persona en la formación del profesorado. Revista Interuniversitaria de Formación del Profesorado, 68, pp. 83-101.

Lakoff, G. y Johnson, M. (1986). Metáforas de la vida cotidiana. Madrid: Cátedra.

Lasky, S. (2000). The Cultural and Emotional Politics of Teacher-Parent Interactions. Teaching and Teacher Education, 16(8), pp. 843-860.

http://dx.doi.org/10.1016/S0742-051X(00)00030-5

LeAVy, A.M.; MCSorley, F.A. y Boté, L.A. (2007). An examination of what metaphor construction reveals about the evolution of preservice teachers' beliefs about teaching and learning. Teaching and Teacher Education, 23, pp. 1217-1233.

http://dx.doi.org/10.1016/j.tate.2006.07.016

López-Ramírez, E.O. (2009). Las emociones, México: Trillas.

Magnusson, S.; Krajcik, J. y Borko, H. (1999). Nature, sources, and development of the PCK for science teaching. En J. Gess-Newsome y N.G. Lederman (Eds.). Examining pedagogical content knowledge. Dordrecht: Kluwer, pp. 95-132.

Manassero, M.A. (2013). Emociones: del olvido a la centralidad en la explicación del comportamiento. En V. Mellado, L.J. Blanco, A.B. Borrachero y J. Cárdenas (Eds.). Las emociones en la enseñanza y el aprendizaje de las ciencias y las matemáticas (Vol. I). Badajoz: DEPROFE.UEX, pp. 3-18.

Marbá, A. y Márquez, C. (2010). ¿Qué opinan los estudiantes de las clases de ciencias? Un estudio transversal de sexto de primaria a cuarto de ESO. Enseñanza de las Ciencias, 28(1), pp. 19-30.

Marina, J.A. (2004). La inteligencia fracasada. Barcelona: Anagrama.

McCaughtry, N. (2005). Elaborating pedagogical content knowledge: what it means to know students and think about teaching. Teachers and Teaching: Theory and Practice, 11(4), pp. 379-395. http://dx.doi.org/10.1080/13450600500137158

McLeod, D.B. (1989). Beliefs, attitudes, and emotions: new view of affect in mathematics education. En D.B. McLeod y V.M. Adams (Eds.). Affect and mathematical problem solving: A new perspective. New York: Springer-Verlang, pp. 245-258.

Mellado, L.; Bermejo, M.L. y Mellado, V. (2012). Personal metaphors of prospective secondary economics and science teachers. Asia Pacific Journal of Teacher Education, 40(4), pp. 395-408. http://dx.doi.org/10.1080/1359866X.2012.724658 
Mellado, L.; Bermejo, M.L.; Fajardo, M.I. y Luengo, M.R. (2013). Las emociones en las metáforas personales de futuros profesores de Ciencias, de Economía y de Psicopedagogía. En V. Mellado, L.J. Blanco, A.B. Borrachero y J.A. Cárdenas (Eds.). Las Emociones en la Enseñanza y el Aprendizaje de las Ciencias y las Matemáticas. Badajoz: DEPROFE, pp. 417-438.

Mellado, V. (2003). Cambio didáctico del profesorado de ciencias experimentales y filosofía de la ciencia. Enseñanza de las Ciencias, 21(3), pp. 343-358.

Mellado, V. (2011). Formación del profesorado y buenas prácticas: el lugar de la innovación y la investigación educativa. En P. Cañal (coord.). Biología y Geología. Investigación, innovación y buenas prácticas. Barcelona: Ed. GRAO, pp. 9-29.

Mellado, V.; Blanco, L.J.; Borrachero, A.B. y Cárdenas, J. (2013). Las emociones en la enseñanza y el aprendizaje de las ciencias y las matemáticas (Vol. I y II). Badajoz: DEPROFE. <http://www. eweb.unex.es/eweb/dcem/novedades.htm>.

Melo, L.; Cañada, F.; Mellado, V.; Díaz, M. y Melo, D. (2013). La evaluación como componente de análisis del conocimiento didáctico del contenido en el caso del campo eléctrico. Campo Abierto, 32(2), pp. 173-197.

Mora, F. (2008). El reloj de la sabiduría. Tiempos y espacios en el cerebro humano. Madrid: Alianza Editorial.

Olitshy, S. y Milne, C. (2012). Understanding engagement in science education: The psychological and the social. En B.J. Fraser, K.G. Tobin y C.J. McRobbie (Eds.). Second International Handbook of Science Education. Dordrecht: Springer, pp. 19-33.

Oosterheert, I.E. y Vermunt, J.D. (2001). Individual differences in learning to teach: relating cognition, regulation an affect. Learning and Instruction, 11, pp. 133-156.

http://dx.doi.org/10.1016/S0959-4752(00)00019-0

Otero, M.R. (2006). Emociones, Sentimientos y Razonamientos en Didáctica de las Ciencias. Revista Electrónica de Investigación en Educación en Ciencias, 1(1). <http://www.exa.unicen.edu.ar/ reiec/?q=es/anio 1 num $1>$.

Padilla, K. y VAn Driel, J.H. (2012). Relationships among cognitive and emotional dimensions of teaching quantum chemistry at university level. Educación Química, 23(E2), pp. 311-326.

Park, S. y Oliver, J.S. (2008). Revisiting the Conceptualisation of Pedagogical Content Knowledge (PCK): PCK as a Conceptual Tool to Understand Teachers as Professionals. Research in Science Education, 38, pp. 261-284.

http://dx.doi.org/10.1007/s11165-007-9049-6

Peme, C.; Mellado, V.; De Longhi, A.L.; Argañaraz, M.R. y Ruiz, C. (2008). La interacción entre las concepciones y la práctica de una profesora de Ciencias Naturales de secundaria con experiencia: Un programa longitudinal de desarrollo profesional basado en la reflexión orientada colaborativa. TED: Tecne, Episteme y Didaxis, 24, pp. 75-98.

Pérez, A. y de Pro, A. (2013). Estudio demoscópico de lo que sienten y piensan los niños y adolescentes sobre la enseñanza formal de las ciencias. En V. Mellado, L.J. Blanco, A.B. Borrachero y J.A. Cárdenas (Eds.). Las Emociones en la Enseñanza y el Aprendizaje de las Ciencias y las Matemáticas. Badajoz, España: DEPROFE, pp. 495-520.

Pintrich, P.R.; Marx, R.W. y Boyle, R.A. (1993). Beyond cold conceptual change: the role of motivational beliefs and classroom contextual factors in the process of conceptual change. Review of Educational Research, 63(2), pp. 167-199.

http://dx.doi.org/10.3102/00346543063002167

Porlán, R.; Martín del Pozo, R.; Rivero, A.; Harres, J.; Azcárate, P. y Pizzato, M. (2010). El cambio del profesorado de ciencias I: marco teórico y formativo. Enseñanza de las Ciencias, 28(1), pp. 31-46. 
Pro, A. De (2006). Perfil de la «Reforma LOGSE» y perfil de uso: los fundamentos de los proyectos curriculares de Física y Química en centros de secundaria. Enseñanza de las Ciencias, 24(3), pp. 337-356.

Pro, A. de; Sánchez, G. y Valcárcel, M.V. (2008). Análisis de los libros de texto de física y química en el contexto de la reforma LOGSE. Enseñanza de las Ciencias, 26(2), pp. 193-210.

Punset, E. (2010). Viaje a las emociones. Barcelona: destino.

Ritchie, S.M.; Tobin, K.; Hudson, P.; Roth, W.M. y Mergard, V. (2011). Reproducing successful rituals in bad times: exploring emotional interactions of a new science teacher. Science Education, 95(4), pp. 745-765. http://dx.doi.org/10.1002/sce.20440

Rocard, M.; Csermely, P.; Jorde, D.; Lenzen, D.; Walwerg-Henriksson, H. y Hemmo, V. (2007). Science Education Now: A Renewed Pedagogy for the Future of Europe. European Commission, Community Research.

Rosiek, J. y Beghetto, R.A. (2011). Emotional scaffolding and imaginative dimensions of teaching and learning. En P.A. Schtz y M. Zembylas (Eds.). Advances in teacher emotion research. Dordrecht: Springer, pp. 175-194.

Schutz, P.A. y Zembylas, M. (2011) (Eds.). Advances in teacher emotion research. Dordrecht: Springer.

Shulman, L.S. (1986). Those who understand: knowledge growth in teaching. Educational Researcher, 15(2), pp. 4-14.

http://dx.doi.org/10.3102/0013189X015002004

Shulman, L.S. (1987). Knowledge and Teaching: Foundations of the New Reform, Harvard Educational Review, 57(1), pp. 1-22.

Shulman, L.S. (2012). Keynote at the PCK Summit. Colorado Springs, octubre 20-25 de 2012. <http://pcksummit.bscs.org/>.

Sutton, C. (1997). Ideas sobre la ciencia e ideas sobre el lenguaje. Alambique, 12, pp. 8-32.

Thagard, P. (2008). Conceptual change in the history of science: life, mind and disease. En S. Vosniadou (Ed.): International Handbook of Research on Conceptual Change. New York: Routledge, pp. 374-387.

Thomas, L. y Ritchie, S.M. (2012). Positive Emotional Responses to Hybridised Writingabout a Socio-Scientific Issue. Research in Science Education, 42(1), pp. 25-49. http://dx.doi.org/10.1007/s11165-011-9255-0

Tовіn, K. (2010). Reproducir y transformar la didáctica de las ciencias en un ambiente colaborativo. Enseñanza de las Ciencias, 28(3), pp. 301-313.

Tobin, K., Tippins, D.J. y Gallard, A.J. (1994). Research on instructional strategies for teaching science. En D. Gabel (Ed.). Handbook of Research on Science Teaching and Learning. New York: MacMillan, pp. 3-44.

VÁzquez, A. y Manassero, M.A. (2007). En defensa de las actitudes y emociones en la educación científica (I): evidencias y argumentos generales. Revista Eureka sobre Enseñanza y Divulgación de las Ciencias, 4(2), pp. 247-271.

VÁzQuez, A. y Manassero, M.A. (2008). El declive de las actitudes hacia la ciencia de los estudiantes: un indicador inquietante para la educación científica. Revista Eureka, 5(3), pp. 274-292.

VÁzquez, A. y Manassero, M.A. (2011). El descenso de las actitudes hacia la ciencia de chicos y chicas en la educación obligatoria. Ciência y Educação, 17(2), pp. 249-268.

http://dx.doi.org/10.1590/S1516-73132011000200001

Weiner, B. (1986). An attributional theory of motivation and emotions. New York: Springer. http://dx.doi.org/10.1007/978-1-4612-4948-1 
White, G. (1993). Emotions inside out: the anthropology of affect. En M. Lewis y J. Haviland (Eds.). Handbook of emotions. New York: Guildford Press.

Zembylas, M. (2002). Constructing genealogies of teachers' emotions in science teaching. Journal of Research in Science Teaching, 39(1), pp. 79-103.

http://dx.doi.org/10.1002/tea.10010

Zembylas, M. (2004). Emotion metaphors and emotional labor in science teaching. Science Education, 88(3), pp. 301-324.

http://dx.doi.org/10.1002/sce.10116

Zembylas, M. (2007). Emotional ecology: The intersection of emotional knowledge and pedagogical content knowledge in teaching. Teaching and Teacher Education, 23(4), pp. 355-367.

http://dx.doi.org/10.1016/j.tate.2006.12.002 


\title{
Emotions in Science teaching
}

\author{
Vicente Mellado, ,A. Belén Borrachero, María Brígido, Lina V. Melo, M.Antonia Dávila, Florentina Cañada, \\ M. Carmen Conde, Emilio Costillo, Javier Cubero, Rocío Esteban, Guadalupe Martínez, Constantino Ruiz, Jesús Sánchez \\ Departamento de Didáctica Ciencias Experimentales y Matemáticas. Universidad de Extremadura. \\ vmellado@unex.es \\ Andoni Garritz \\ Facultad de Química, Universidad Nacional Autónoma de México. México DF. \\ Lucía Mellado \\ Departamento de Economía de La Empresa y Contabilidad. Facultad Ciencias Económicas y Empresariales. UNED. \\ Bartolomé Vázquez, Roque Jiménez \\ Departamento de Didáctica de las Ciencias y Filosofía. Universidad de Huelva. \\ M. Luisa Bermejo \\ Departamento de Psicología y Antropología. Universidad de Extremadura.
}

After years of neglect, emotions are beginning to form part of the Science education's research agenda with recognition of their linkage with the teaching and learning of specific knowledge. For instance, teachers and pupils are constantly required to make decisions in class and, in this process, emotions plays a central role. However, it is by no means our intention in studying emotions to replace rational reductionism with emotional reductionism, but rather to accept emotions and reason are complementary in nature.

The study of emotions in the context of Science teaching can provide data that will contribute to making teachers and their pupils aware of the importance of their emotions in the learning process, and to accept that they can be emotionally vulnerable. The data can also contribute to setting up programs of meta-emotional and meta-cognitive intervention in teacher education that are oriented towards prospective teachers - and subsequently their own pupils when they themselves are teaching- recognizing, and then controlling and self-regulating their emotions.

Although initially emotions and the affective domain were not regarded as part of the pedagogical content knowledge, in recent years many authors have argued that they are actually an essential part of this knowledge in whatever modality.

Various studies have indicated that prospective teachers emotionally have very positive memories of Science subjects when they were in primary school. This does not totally apply, however, to their memories of their secondary school years. Instead, while they recall their emotions in learning Biology and Geology to have been very positive, those in learning Physics and Chemistry were mostly negative. A surprising finding was that they attributed the cause of their positive emotions primarily to their teachers, as against the content of the subject or themselves as learners. Contrarily, they attributed the cause of their negative emotions more to the content and to themselves as learners than to their teachers.

For both primary and secondary prospective teachers, the emotions they recalled as learners of the different Science subjects transferred to those they experienced when teaching these subjects during their practicums, although these latter emotions were generally more positive. Another result was that women reported their emotions, whether positive or negative, as being more intense than men did, and they had more negative emotions when teaching Physics and similar content.

Regarding other variables, self-efficacy, i.e., belief in their own competence, was found to influence their emotions about teaching the content which arouse more negative emotions, such as Physics. In particular, the greater the selfefficacy for teaching this type of content, the more positive or less negative the emotions felt about teaching that content.

Emotions also influence both the professional development of Science teachers and the success or failure of the implementation of education reforms. Teachers will only put into practice changes which compensate them affectively, and for which they feel they are supported and understood by their professional environment.

Negative emotions often constitute an obstacle to learning and teaching Science. In schools, there has often been excessive use of the Science teaching approach of transmitting a set of finished and indisputable truths that are distant not only from the pupils' interests and concerns, but also from the emotions that resulted from the construction of knowledge at each particular time in History. This approach to Science teaching generates negative attitudes and emotions towards certain subjects which many pupils carry over, years later, to their professional field, if they take up a teaching career where they have to teach those subjects. This vicious circle has to be broken during initial teacher education. The challenge is to replace negative emotions with positive ones through creative and challenging scientific activities. After all, emotions have made scientific activity an adventure of thought and a profoundly human endeavour throughout History, which has decisively contributed to our understanding of nature and to the progress of mankind. 